\title{
Nonperturbative Description of Deep Inelastic Structure Functions in Light-Front QCD
}

\author{
A. Harindranath ${ }^{a}$, Rajen Kundu ${ }^{a}$, and Wei-Min Zhang ${ }^{b}$ \\ ${ }^{a}$ Saha Institute of Nuclear Physics, 1/AF, Bidhan Nagar, Calcutta 700064 India \\ ${ }^{b}$ Institute of Physics, Academia Sinica, Taipei, Taiwan 11529, ROC
}

(Feb. 5, 1998)

\begin{abstract}
In this paper, we explore the deep inelastic structure functions of hadrons nonperturbatively in an inverse power expansion of the light-front energy of the probe in the framework of light-front QCD. We arrive at the general expressions for various structure functions as the Fourier transform of matrix elements of different components of bilocal vector and axial vector currents on the light-front in a straightforward manner. The complexities of the structure functions are mainly carried by the multi-parton wave functions of the hadrons, while, the bilocal currents have a dynamically dependent yet simple structure on the light-front in this description. We also present a novel analysis of the power corrections based on light-front power counting which resolves some ambiguities of the conventional twist analysis in deep inelastic processes. Further, the factorization theorem and the scale evolution of the structure functions are presented in this formalism by using old-fashioned light-front time-ordered perturbation theory with multi-parton wave functions. Nonperturbative QCD dynamics underlying the structure functions can be explored in the same framework. Once the nonperturbative multi-parton wave functions are known from low-energy light-front QCD, a complete description of deep inelastic structure functions can be realized.
\end{abstract}

PACS numbers: 12.38.-t, 13.85.Hd, 13.88.+e, 11.30.Rd 


\section{INTRODUCTION}

With the recent and planned experiments on polarized and unpolarized structure functions, the field of deep inelastic scattering has entered a new era. New experiments are beginning to provide invaluable information on the so-called "higher twist" (power suppressed) contributions to deep inelastic cross sections, a theoretical understanding of which requires nonperturbative information on the structure of hadrons. To unravel this structure, there is an urgent need to develop nonperturbative theoretical tools which are preferably based on physical intuition and which at the same time employs well-defined field theoretical calculational procedures. Towards this goal, in this work, we propose a new method of calculation of structure functions combining the coordinate space approach based on lightfront current algebra techniques and the momentum space approach based on Fock space expansion methods in light-front theory in a Hamiltonian QCD framework.

To get an intuitive picture of deep inelastic scattering in field theory, it is extremely helpful to keep close contact with parton ideas. However, partons were originally introduced as collinear, massless, on-mass shell objects. In reality, the QCD governed interacting partons should not be collinear and massless. The question, then, is can one generalize this concept and introduce field theoretic partons as non-collinear and massive (in the case of quarks) but still on-mass shell objects in interacting field theory? Nonperturbative light-front Hamiltonian description of composite systems which utilizes many body wave functions for the constituents allows us to precisely achieve this goal.

Now, the problem is how to introduce many body (or multi-parton) wave functions in the description of deep inelastic structure functions. An attractive possible avenue is provided by the BJL (high energy) expansion of scattering amplitudes together with the use of lightfront current algebra. This is essentially a nonperturbative approach where the expansion parameter is the inverse of the light-front energy of the probe (in the present case, the virtual photon). In this approach one can arrive at expressions for various structure functions as the Fourier Transform (in the light-front longitudinal direction) of matrix elements of different components of bilocal vector and axial vector currents in light-front field theory.

In the standard approach to deep inelastic scattering based on Wilson's Operator Product Expansion (OPE) method which is more mathematical, one considers the problem of renormalizing composite operators. In contrast, in the current algebra approach, products of field operators are evaluated at equal light-front time and have the same form as their free field theory counterparts. Hence in this approach, most of the complexities appear to be carried by the hadronic states. We demonstrate that an expansion of the state allows us to exhibit this complexity manifestly in terms of the multi-parton wave functions, where the constituents are on-mass shell objects with non-vanishing transverse momenta. The nonperturbative nature of the matrix elements relevant for various structure functions are thus directly translated into the language of multi-parton wave functions. The structure functions, then, can be conveniently evaluated once the nonperturbative wave functions renormalized at scale $Q$ are known. At present major efforts are underway to evaluate these wave functions.

With the advent of QCD, the current algebra approach which originally lead to the prediction of scaling without introducing the concept of partons was altogether abandoned in

favor of the OPE method, primarily because it was known that the canonical manipulations 
that lead to many of the current algebra predictions are invalidated in perturbation theory which gives rise to logarithmic corrections. The only exceptions were sum rules protected by conservation laws. Obviously, what was missing in the current algebra approach was the realization that the matrix elements relevant for deep inelastic scattering are those renormalized at the physical scale $Q$. Thus one of our major problem is whether we can consistently carry out renormalization procedure. In a subsequent paper [1], we shall demonstrate that in the case of leading order structure functions the scaling violations of perturbation theory can be successfully addressed by replacing the hadron target by a dressed parton target in the matrix element and carrying out a well-defined perturbative expansion which closely follows the techniques of (light-front) time-ordered perturbation theory [2 0 . Here, we will show that we can address the issues of factorization and scale evolution in the case of a hadron target by separating the soft and hard parts of the multi-parton wave functions. Furthermore, the nonperturbative contributions to deep inelastic structure functions can be addressed within the same framework by incorporating the newly developed light-front renormalization group approach to nonperturbative QCD [5 [7]. Therefore, a unified lightfront description of the perturbative and nonperturbative QCD underlying the deep inelastic structure functions can be realized.

The paper is organized as follows. In Sec. II, a brief overview of deep inelastic structure functions is presented. In Sec. III, we discuss within light-front QCD how the deep inelastic structure functions are nonperturbatively related to the bilocal operators in light-front current algebra through an inverse power expansion of light-front energy of the probe. In Sec. IV, we determine the structure functions in terms of the matrix elements of the light-front bilocal vector and axial vector currents separated only in the longitudinal direction. The operator structures involved in the structure functions are explored and the complexities of structure functions are analyzed in Sec. V, where a scheme to evaluate the hard and soft contributions to deep inelastic structure functions in the light-front time-ordering Hamiltonian formalism is proposed. Meanwhile, the concept of twist is examined and redefined on the light-front, which removes some ambiguities in previous works. In Sec. VI, we re-derive

sum rules the structure functions obey and discuss their physical implications. Finally a summary is given in Sec. VII.

\section{A BRIEF OVERVIEW ON DEEP INELASTIC STRUCTURE FUNCTIONS}

We begin with a brief review of the basic ingredients of lepton-nucleon deep inelastic scattering (DIS):

$$
e(k)+h(P) \longrightarrow e(k-q)+X(P+q) .
$$

The cross section for the above scattering process is given by

$$
\frac{d \sigma}{d \Omega d E^{\prime}}=\frac{1}{2 M} \frac{\alpha^{2}}{q^{4}} \frac{E^{\prime}}{E} L_{\mu \nu} W^{\mu \nu},
$$

where $E\left(E^{\prime}\right)$ is the energy of the incoming (outgoing) lepton, $L_{\mu \nu}$ is the leptonic matrix element, 


$$
\begin{aligned}
L_{\mu \nu} & =\frac{1}{2} \sum_{s^{\prime}}\left[\bar{u}(k, s) \gamma_{\mu} u\left(k^{\prime}, s^{\prime}\right) \bar{u}\left(k^{\prime}, s^{\prime}\right) \gamma_{\nu} u(k, s)\right] \\
& =2\left(k_{\mu}^{\prime} k_{\nu}+k_{\nu}^{\prime} k_{\mu}\right)-2 g_{\mu \nu} k \cdot k^{\prime}-2 i \epsilon_{\mu \nu \rho \sigma} q^{\rho} s^{\sigma},
\end{aligned}
$$

and $W^{\mu \nu}$ the hadronic tensor which contains all the hadronic dynamics involved in DIS process,

$$
W^{\mu \nu}=\frac{1}{4 \pi} \int d^{4} \xi e^{i q \cdot \xi}\left\langle P S\left|\left[J^{\mu}(\xi), J^{\nu}(0)\right]\right| P S\right\rangle,
$$

where $P$ and $S$ are the target four-momentum and polarization vector respectively $\left(P^{2}=\right.$ $\left.M^{2}, S^{2}=-M^{2}, S \cdot P=0\right), q$ is the virtual-photon four momentum, and $J^{\mu}(x)=$ $\sum_{\alpha} e_{\alpha} \bar{\psi}_{\alpha}(x) \gamma^{\mu} \psi_{\alpha}(x)$ the electromagnetic current with quark field $\psi_{\alpha}(x)$ carrying the flavor index $\alpha$ and the charge $e_{\alpha}$.

The above hadronic tensor can be decomposed into independent Lorentz invariant functions:

$$
\begin{aligned}
W^{\mu \nu}= & \left(-g^{\mu \nu}+\frac{q^{\mu} q^{\nu}}{q^{2}}\right) W_{1}\left(x, Q^{2}\right)+\left(P^{\mu}-\frac{\nu}{q^{2}} q^{\mu}\right)\left(P^{\nu}-\frac{\nu}{q^{2}} q^{\nu}\right) W_{2}\left(x, Q^{2}\right) \\
& -i \epsilon^{\mu \nu \lambda \sigma} q_{\lambda}\left[S_{\sigma} W_{3}\left(x, Q^{2}\right)+P_{\sigma} S \cdot q W_{4}\left(x, Q^{2}\right)\right] \\
= & \frac{1}{2}\left(g^{\mu \nu}-\frac{q^{\mu} q^{\nu}}{q^{2}}\right) F_{L}\left(x, Q^{2}\right)+\left[P^{\mu} P^{\nu}-\frac{\nu}{q^{2}}\left(P^{\mu} q^{\nu}+P^{\nu} q^{\mu}\right)+g^{\mu \nu} \frac{\nu^{2}}{q^{2}}\right] \frac{F_{2}\left(x, Q^{2}\right)}{\nu} \\
& -i \epsilon^{\mu \nu \lambda \sigma} \frac{q_{\lambda}}{\nu}\left[S_{\sigma L} g_{1}\left(x, Q^{2}\right)+S_{\sigma T} g_{T}\left(x, Q^{2}\right)\right] .
\end{aligned}
$$

The dimensionless functions

$$
F_{L}\left(x, Q^{2}\right)=2\left[-W_{1}+\left[M^{2}-\frac{(P . q)^{2}}{q^{2}}\right] W_{2}\right]
$$

and

$$
F_{2}\left(x, Q^{2}\right)=\nu W_{2}\left(x, Q^{2}\right)
$$

are the so-called unpolarized structure functions measured from the unpolarized target and

$$
g_{1}\left(x, Q^{2}\right)=\nu\left[W_{3}\left(x, Q^{2}\right)+\nu W_{4}\left(x, Q^{2}\right)\right]
$$

and

$$
g_{T}\left(x, Q^{2}\right)=g_{1}\left(x, Q^{2}\right)+g_{2}\left(x, Q^{2}\right)=\nu W_{3}\left(x, Q^{2}\right),
$$

are the longitudinal and transverse polarized structure functions. While, $x=\frac{Q^{2}}{2 \nu}$ is the famous Bjorken scaling variable, $Q^{2}=-q^{2}$ the momentum transfer carried by the virtual photon and $\nu=P \cdot q$ the energy transfer. The longitudinal and transverse polarization vector component are given by

$$
S_{\mu L}=S_{\mu}-S_{\mu T} \quad, \quad S_{\mu T}=S_{\mu}-P_{\mu} \frac{S \cdot q}{\nu} .
$$


These structure functions provide a probe to explore various aspects of the intrinsic structure of hadrons. It may be worth noting that in the literature, $g_{1}$ and $g_{2}$ are usually used to characterize the longitudinal and transverse polarized structure functions. However, $g_{2}$ is not really a transverse polarized structure function. It also has no clear physical interpretation. Only $g_{T}$ which can be directly measured when the target is polarized along the transverse direction characterizes the full information on the transverse polarization structure.

The early SLAC experiment discovered that the structure function $F_{2}$ depends only on the Bjorken scaling variable $x$, and is independent of momentum transfer $Q^{2}$. This discovery lead to the parton picture proposed first by Feynman [8] in which the constituents in hadrons can be treated as point-like free particles, i.e., the partons. Of course, the picture of point-like and non-interacting partons is certainly over-simplified. As it is well known now, the fundamental constituents (i.e., quarks and gluons) inside hadrons are indeed strongly interacting with each other and are governed by the fundamental QCD theory. Only in the limit of very large $Q^{2}$, the asymptotic freedom feature of QCD makes the quarks and gluons behave as point-like and weakly interacting partons. The scale invariance of $F_{2}$ is violated for finite values of $Q^{2}$. For the past twenty-years, QCD investigations on deep inelastic structure functions are mainly concentrated on the behavior of the scaling violation of these structure functions, which is believed to be the best way to test QCD as the fundamental theory of the strong interaction.

Among these investigations, two methods have dominated the whole research topic: the OPE method [9] and the QCD improved (or field theoretic) parton model based on the factorization scheme [10]. The structure functions have been extensively explored either in terms of its moments in the language of $\mathrm{OPE}$ or in terms of diagrammatic calculation in the language of QCD improved parton model. Both the methods have made great success in understanding the scale evolution of the structure functions within the perturbative QCD domain. Although the deep inelastic scatterings provide a novel way to separate the physics of perturbative and nonperturbative QCD dynamics and allow the exploration of high energy behavior of the constituents inside the hadrons, the structure functions themselves, however, are still dominated by the nonperturbative strong interaction dynamics. A complete understanding of hadrons crucially depends on our understanding of nonperturbative QCD. The OPE method addresses the structure functions in terms of their moments, which naturally separates the short-distance and long-distance dynamics but it also makes the nonperturbative dynamics of long-distance physics more complicated in terms of the moments. On the other hand, the QCD improved parton model was built in the framework of perturbation theory with the assumption of collinearity, which simplifies the perturbative QCD treatment but it is also unclear how to explore nonperturbative QCD dynamics because the nonperturbative QCD dynamics is mainly determined by the non-collinear motion of the low energy quarks and gluons.

In the next section we shall use an inverse power expansion of the light-front energy $q^{-}$of the virtual photon (the extended BJL theorem on the light-front) to extract these deep inelastic structure functions. This approach was originally proposed to study DIS sum rules protected by conservation laws in the pre-QCD era [11]. Here, we shall extend this approach to QCD without recourse to perturbation theory. Therefore it is essentially a direct nonperturbative QCD description. The resulting structure functions are directly 
expressed in terms of the hadronic matrix elements of light-front bilocal vector and axial vector currents, where the currents have a relatively simple structure although they are dynamically dependent. All the hadron dynamics (including both the perturbative and nonperturbative dynamics) reside in the multi-parton hadronic wave functions. This is a description very different from that of the OPE method and the QCD improved parton model.

\section{AN EXPANSION IN INVERSE POWER OF LIGHT-FRONT ENERGY OF THE VIRTUAL PHOTON}

The inverse power expansion of the virtual photon light-front energy $q^{-}$is applied to forward scattering amplitudes. Explicitly, as it is well known, the hadronic tensor is related to the forward virtual-photon hadron Compton scattering amplitude:

$$
W^{\mu \nu}=\frac{1}{2 \pi} \operatorname{Im} T^{\mu \nu}
$$

with

$$
\begin{gathered}
T^{\mu \nu}=i \int d^{4} \xi e^{i q \cdot \xi}\left\langle P S\left|T\left(J^{\mu}(\xi) J^{\nu}(0)\right)\right| P S\right\rangle \\
=\left(-g^{\mu \nu}+\frac{q^{\mu} q^{\nu}}{q^{2}}\right) T_{1}\left(x, Q^{2}\right)+\left(p^{\mu}-\frac{\nu}{q^{2}} q^{\mu}\right)\left(p^{\nu}-\frac{\nu}{q^{2}} q^{\nu}\right) T_{2}\left(x, Q^{2}\right) \\
\quad-i \epsilon^{\mu \nu \lambda \sigma} q_{\lambda}\left[S_{\sigma} T_{3}\left(x, Q^{2}\right)+P_{\sigma} S \cdot q T_{4}\left(x, Q^{2}\right)\right] .
\end{gathered}
$$

Using the optical theorem, we have

$$
T_{i}\left(x, Q^{2}\right)=2 \int_{-\infty}^{\infty} d q^{\prime+} \frac{W_{i}\left(x^{\prime}, Q^{2}\right)}{q^{+}-q^{+}}, \quad i=1,2,3,4 .
$$

Then, as we shall see, the structure functions are connected with the light-front bilocal currents through the $1 / q^{-}$expansion of $T^{\mu \nu}$.

An expansion of $T^{\mu \nu}$ in terms of $1 / q^{-}$was originally proposed by Jackiw et al. [11] based on BJL theorem. The general expansion in $1 / q^{-}$is given by

$$
T^{\mu \nu}=-\sum_{n=0}^{\infty}\left(\frac{1}{q^{-}}\right)^{n+1} \int d \xi^{-} d^{2} \xi_{\perp} e^{i q \cdot \xi}\left\langle P S\left|\left[\left(i \partial_{\xi}^{-}\right)^{n} J^{\mu}(\xi), J^{\nu}(0)\right]_{\xi^{+}=0}\right| P S\right\rangle
$$

where $q^{-}=q^{0}-q^{z}$, the light-front energy of the virtual photon, and $\partial^{-}=2 \frac{\partial}{\partial \xi^{+}}$is a light-front time derivative. The light-front coordinates of the space-time are defined by

$$
\xi^{ \pm}=\xi^{0} \pm \xi^{3}, \quad \xi_{\perp}^{i}=\xi^{i}(i=1,2) .
$$

The above expansion shows that the time-ordered matrix element can be expanded in terms of an infinite series of equal light-front time commutators.

For large $Q^{2}$ and large $\nu$ limits in DIS, theoretically without loss of generality we can always select a Lorentz frame such that the light-front energy $q^{-}$of the virtual photon becomes very large. Then, only the leading term in the above expansion is dominant, i.e., 


$$
T^{\mu \nu} \stackrel{\text { large }}{=} q^{-}-\frac{1}{q^{-}} \int d \xi^{-} d^{2} \xi_{\perp} e^{i q \cdot \xi}\left\langle P S\left|\left[J^{\mu}(\xi), J^{\nu}(0)\right]_{\xi^{+}=0}\right| P S\right\rangle
$$

As a result, the leading contribution to the deep inelastic structure functions is determined by the light-front current algebra. The light-front current commutator can be computed directly and exactly from QCD (where QCD should be quantized on the light-front time surface $\xi^{+}=$ $\xi^{0}+\xi^{3}=0$ with the light-front gauge $A_{a}^{+}=0$ [2, 3] $)$. Hence, all the subsequent derivations are exact within the light-front QCD and without further assumptions or approximations of the collinear and massless partons that were used in the previous derivations [9, 10].

Explicitly, the basic commutation relation on light-front is

$$
\left\{\psi_{+}(x), \psi_{+}^{\dagger}(y)\right\}_{x^{+}=y^{+}}=\Lambda^{+} \delta\left(x^{-}-y^{-}\right) \delta^{2}\left(x_{\perp}-y_{\perp}\right),
$$

which is exact in the full QCD theory [3], where $\psi_{+}(x)$ is called the dynamical component of fermion field on the light-front:

$$
\psi(x)=\psi_{+}(x)+\psi_{-}(x), \quad \psi_{ \pm}(x)=\Lambda^{ \pm} \psi(x), \quad \Lambda^{ \pm}=\frac{1}{2} \gamma^{0} \gamma^{ \pm}
$$

The minus component $\psi_{-}(x)$ is determined from $\psi_{+}(x)$ as a result of Dirac equation:

$$
\psi_{-}(x)=\frac{1}{i \partial^{+}}\left(i \alpha_{\perp} \cdot D_{\perp}+\beta m_{q}\right) \psi_{+}(x) .
$$

Here we have already used the light-front gauge $A_{a}^{+}=0$, and $D_{\perp}=\partial_{\perp}-i g A_{\perp}$ is the transverse component of the covariant derivative, $\alpha_{\perp}^{i}=\gamma^{0} \gamma^{i}, \beta=\gamma^{0}$.

Because of the above special property of quark (or more generally fermion) field on the light-front, the light-front current explicitly depends on interaction of the theory, which is very different from the usual equal-time formulation. In other words, the fundamental interaction is manifested explicitly in the light-front current commutators.

From Eqs.(3.7) and (3.9), we have

$$
\left\{\psi_{+}(x), \psi_{-}^{*}(y)\right\}_{x^{+}=y^{+}}=\frac{\Lambda^{+}}{4 i} \epsilon\left(x^{-}-y^{-}\right)\left[i \alpha_{\perp} \cdot D_{\perp}^{*}-\beta m\right] \delta^{2}\left(x_{\perp}-y_{\perp}\right) .
$$

Thus, after a tedious but straightforward calculation, one can find that

$$
\begin{array}{r}
{\left[J^{+}(x), J^{-}(y)\right]_{x^{+}=y^{+}}=\sum_{\alpha} e_{\alpha}^{2}\left\{\partial_{x}^{+}\left[-\frac{1}{2} \epsilon\left(x^{-}-y^{-}\right) \delta^{2}\left(x_{\perp}-y_{\perp}\right) V_{\alpha}^{-}(x \mid y)\right]\right.} \\
+\partial_{x}^{i}\left[\frac { 1 } { 2 } \epsilon ( x ^ { - } - y ^ { - } ) \delta ^ { 2 } ( x _ { \perp } - y _ { \perp } ) \left[V_{\alpha}^{i}(x \mid y)\right.\right. \\
\left.\left.\left.+i \epsilon^{i j} A_{\alpha}^{j}(x \mid y)\right]\right]- \text { h.c. }\right\}
\end{array}
$$

where $V_{\alpha}^{\mu}$ and $A_{\alpha}^{\mu}$ are defined as the bilocal vector and axial vector currents:

$$
\begin{aligned}
& V_{\alpha}^{\mu}(x \mid y)=\bar{\psi}_{\alpha}(x) \gamma^{\mu} \psi_{\alpha}(y) \\
& A_{\alpha}^{\mu}(x \mid y)=\bar{\psi}_{\alpha}(x) \gamma^{\mu} \gamma_{5} \psi_{\alpha}(y)
\end{aligned}
$$


As we can see, the light-front current commutators are very different from the equaltime current commutators. Here the commutator is indeed given by terms involving spatial derivatives. These space-derivatives come from the non-locality of $\psi_{-}(x)$ on the light-front. In the equal-time formulation, there is no such nonlocality to the fermion field. Therefore one cannot derive such a commutator from the naive canonical equal-time commutations. As we will soon see in the next section it is these spatial derivatives that lead to the simple expressions of the structure functions in terms of bilocal current matrix elements. This is an essential feature in the present approach that make the light-front current algebra specially useful in the exploration of the deep inelastic structure functions.

The commutators for other current components can also be found straightforwardly. For example,

$$
\begin{aligned}
{\left[J^{+}(x),\right.} & \left.J^{i}(y)\right]_{x^{+}=y^{+}}=\sum_{\alpha} e_{\alpha}^{2}\left\{\partial_{x}^{+}\left[-\frac{1}{2} \epsilon\left(x^{-}-y^{-}\right) \delta^{2}\left(x_{\perp}-y_{\perp}\right) V_{\alpha}^{i}(x \mid y)\right]\right. \\
& \left.+\partial_{x}^{j}\left[\frac{1}{2} \epsilon\left(x^{-}-y^{-}\right) \delta^{2}\left(x_{\perp}-y_{\perp}\right)\left(g^{i j} V_{\alpha}^{+}(x \mid y)+i \epsilon^{i j} A_{\alpha}^{+}(x \mid y)\right)\right]-\text { h.c. }\right\} .
\end{aligned}
$$

Thus, one can use Eq.(3.11) to extract the structure functions and then use Eq.(3.14) to make a consistency check.

Now, the Compton scattering amplitude in the large $q^{-}$limit can be immediately expressed in terms of the hadronic matrix elements of the bilocal vector and axial vector currents. For example, the $(+-)$ component $T^{+-}$is given by

$$
\begin{aligned}
& T^{+-} \stackrel{\text { large }}{=} q^{-}-\frac{1}{q^{+}} \int d \xi^{-} e^{i q^{+} \xi^{-} / 2} \epsilon(\xi)\langle P S| \sum_{\alpha} e_{\alpha}^{2}\left\{\frac{i}{2} q^{+} V_{\alpha}^{-}\left(\xi^{-} \mid 0\right)\right. \\
& \left.-\frac{i}{2} q_{\perp}^{i}\left[V_{\alpha}^{i}\left(\xi^{-} \mid 0\right)+i \epsilon^{i j} A_{\alpha}^{j}\left(\xi^{-} \mid 0\right)\right]\right\}- \text { h.c. }|P S\rangle .
\end{aligned}
$$

The above result shows that the bilocal vector and axial vector currents entering in $T^{\mu \nu}$ are separated only in the longitudinal direction $\xi^{-}=\xi^{0}-\xi^{3}$. This property naturally leads to the well-known scaling behavior of the structure functions when we ignore the QCD dynamics at very high $Q^{2}$. In the next section, we will extract explicitly the structure functions in terms of these bilocal current matrix elements.

\section{THE GENERALIZED EXPRESSIONS FOR DEEP INELASTIC STRUCTURE FUNCTIONS}

Now, let us pick up the same $(+-)$ component of the hadronic tensor Eq.(2.5) to find the deep inelastic structure function by comparing with Eq.(3.15) through Eq.(3.3). In the large $q^{-}$limit,

$$
\begin{aligned}
W^{+-}=\frac{1}{2} F_{L}+ & \left(P_{\perp}\right)^{2} \frac{F_{2}}{\nu}-2 P_{\perp} \cdot q_{\perp} \frac{F_{2}}{q^{2}} \\
& +2 i \epsilon^{i j} q_{i}\left[S_{j L} \frac{g_{1}}{\nu}+S_{j T} \frac{g_{T}}{\nu}\right],
\end{aligned}
$$

where $S_{j T}=S_{j}-S^{+} \frac{P_{j}}{P^{+}}$and $S_{j L}=S_{j}-S_{j T}=S^{+} \frac{P_{j}}{P^{+}}$, and $\nu=\frac{1}{2} P^{+} q^{-}$in the large $q^{-}$limit. We introduce the form factors for the bilocal current matrix elements, 


$$
\begin{gathered}
\left\langle P S\left|V_{\alpha}^{\mu}(\xi \mid 0)-V_{\alpha}^{\mu}(0 \mid \xi)\right| P S\right\rangle=P^{\mu} \bar{V}_{1 \alpha}\left(P^{2}, \xi \cdot P\right)+\xi^{\mu} \bar{V}_{2 \alpha}\left(P^{2}, \xi \cdot P\right) \\
\left\langle P S\left|A_{\alpha}^{\mu}(\xi \mid 0)+A_{\alpha}^{\mu}(0 \mid \xi)\right| P S\right\rangle=S^{\mu} \bar{A}_{1 \alpha}\left(P^{2}, \xi \cdot P\right)+P^{\mu} \xi \cdot S \bar{A}_{2 \alpha}\left(P^{2}, \xi \cdot P\right) \\
+\xi^{\mu} S \cdot \xi \bar{A}_{3 \alpha}\left(P^{2}, \xi \cdot P\right) .
\end{gathered}
$$

Since, $\xi^{+, \perp}=0$, it follows that the matrix elements of the plus and transverse components of the bilocal current yield the same form factor $\bar{V}_{1 \alpha}$. Using the definition

$$
\epsilon\left(\xi^{-}\right)=-\frac{i}{\pi} \int_{-\infty}^{\infty} \frac{d q^{+}}{q^{+}} e^{i q^{+} \xi^{-} / 2}
$$

we find that

$$
\begin{array}{r}
T^{+-}=-\frac{1}{\pi q^{-}} \int_{-\infty}^{\infty} \frac{d q^{\prime+}}{q^{\prime+}-q^{+}} \int_{-\infty}^{\infty} d \xi^{-} e^{i q^{+} \xi^{-} / 2} \sum_{\alpha} e_{\alpha}^{2}\left\{\frac{1}{2}\left(P^{-} q^{+}-P_{\perp} \cdot q_{\perp}\right) \bar{V}_{1 \alpha}\right. \\
\left.+\frac{1}{2} q^{+} \xi^{-} \bar{V}_{2 \alpha}+\frac{i}{2} \epsilon^{i j} q_{i}\left[S_{j} \bar{A}_{1 \alpha}+P_{j} \frac{S^{+} \xi^{-}}{2} \bar{A}_{2 \alpha}\right]\right\} .
\end{array}
$$

The bilocal current form factors are determined from Eq.(4.2):

$$
\begin{aligned}
\bar{V}_{1 \alpha} & =\frac{1}{P^{+}}\left\langle P S\left|\bar{\psi}_{\alpha}\left(\xi^{-}\right) \gamma^{+} \psi_{\alpha}(0)-\bar{\psi}_{\alpha}(0) \gamma^{+} \psi_{\alpha}\left(\xi^{-}\right)\right| P S\right\rangle \\
& =\frac{1}{P^{i}}\left\langle P S\left|\bar{\psi}_{\alpha}\left(\xi^{-}\right) \gamma^{i} \psi_{\alpha}(0)-\bar{\psi}_{\alpha}(0) \gamma^{i} \psi_{\alpha}\left(\xi^{-}\right)\right| P S\right\rangle, \\
\bar{V}_{2 \alpha} & =\frac{1}{\xi^{-}}\left\langle P S\left|\bar{\psi}_{\alpha}\left(\xi^{-}\right)\left(\gamma^{-}-\frac{P^{-}}{P^{+}} \gamma^{+}\right) \psi_{\alpha}(0)-h . c .\right| P S\right\rangle \\
& =\frac{1}{\xi^{-}}\left\langle P S\left|\bar{\psi}_{\alpha}\left(\xi^{-}\right)\left(\gamma^{-}-\frac{P^{-}}{P^{i}} \gamma^{i}\right) \psi_{\alpha}(0)-h . c .\right| P S\right\rangle, \\
\bar{A}_{1 \alpha} & =\frac{1}{S_{T}^{i}}\left\langle P S\left|\bar{\psi}_{\alpha}\left(\xi^{-}\right)\left(\gamma^{i}-\frac{P^{i}}{P^{+}} \gamma^{+}\right) \gamma_{5} \psi_{\alpha}(0)+h . c .\right| P S\right\rangle, \\
\bar{A}_{2 \alpha} & =\frac{-2}{P^{+} \xi^{-} S}\langle P S| \bar{\psi}_{\alpha}^{i}\left(\xi^{-}\right)\left(\gamma^{i}-\frac{S^{i}}{S^{+}} \gamma^{+}\right) \gamma_{5} \psi_{\alpha}(0)+\text { h.c. }|P S\rangle .
\end{aligned}
$$

Comparing with Eqs.(4.1) and (4.5) through Eq.(3.3), we obtain, with $\eta \equiv \frac{1}{2} P^{+} \xi^{-}$,

$$
\begin{aligned}
\frac{F_{2}\left(x, Q^{2}\right)}{x} & =\frac{1}{4 \pi} \int d \eta e^{-i \eta x} \sum_{\alpha} e_{\alpha}^{2} \bar{V}_{1 \alpha} \\
& =\frac{1}{4 \pi P^{+}} \int d \eta e^{-i \eta x} \sum_{\alpha} e_{\alpha}^{2}\left\langle P S\left|\bar{\psi}_{\alpha}\left(\xi^{-}\right) \gamma^{+} \psi_{\alpha}(0)-\bar{\psi}_{\alpha}(0) \gamma^{+} \psi_{\alpha}\left(\xi^{-}\right)\right| P S\right\rangle \\
& =\frac{1}{4 \pi P_{\perp}^{i}} \int d \eta e^{-i \eta x} \sum_{\alpha} e_{\alpha}^{2}\left\langle P S\left|\bar{\psi}_{\alpha}\left(\xi^{-}\right) \gamma_{\perp}^{i} \psi_{\alpha}(0)-\bar{\psi}_{\alpha}(0) \gamma_{\perp}^{i} \psi_{\alpha}\left(\xi^{-}\right)\right| P S\right\rangle,
\end{aligned}
$$

where the last equality is found for the first time here [12. Its physical interpretation will be given later.

$$
\begin{aligned}
F_{L}\left(x, Q^{2}\right)= & -\frac{q^{+}}{\pi P^{+} q^{-}} \int d \eta e^{-i \eta x} \sum_{\alpha} e_{\alpha}^{2}\left[\left(P^{-}-\frac{P_{\perp}^{2}}{P^{+}}\right) \bar{V}_{1 \alpha}+\xi^{-} \bar{V}_{2 \alpha}\right] \\
= & \frac{P^{+}}{4 \pi}\left(\frac{2 x}{Q}\right)^{2} \int d \eta e^{-i \eta x} \sum_{\alpha} e_{\alpha}^{2}\langle P S| \bar{\psi}_{\alpha}\left(\xi^{-}\right) \\
& \times\left(\gamma^{-}-\frac{P_{\perp}^{2}}{\left(P^{+}\right)^{2}} \gamma^{+}\right) \psi_{\alpha}(0)-\text { h.c. }|P S\rangle
\end{aligned}
$$


where the first equality may be reduced to the same expression obtained by the collinear expansion in the Feynman diagrammatic method up to the order twist-four [14]. But here it is directly obtained in the leading order in the $1 / q^{-}$expansion without involving the concept of twist expansion. Moreover, the polarized structure functions can also be found directly as

$$
\begin{aligned}
g_{1}\left(x, Q^{2}\right) & =\frac{1}{8 \pi} \int d \eta e^{-i \eta x} \sum_{\alpha} e_{\alpha}^{2}\left(\bar{A}_{1 \alpha}+\frac{1}{2} P^{+} \xi^{-} \bar{A}_{2 \alpha}\right) \\
& =\frac{1}{8 \pi S^{+}} \int d \eta e^{-i \eta x} \sum_{\alpha} e_{\alpha}^{2}\left\langle P S\left|\bar{\psi}_{\alpha}\left(\xi^{-}\right) \gamma^{+} \gamma_{5} \psi_{\alpha}(0)+\bar{\psi}(0) \gamma^{+} \gamma_{5} \psi\left(\xi^{-}\right)\right| P S\right\rangle, \\
g_{T}\left(x, Q^{2}\right) & =\frac{1}{8 \pi} \int d \eta e^{-i \eta x} \sum_{\alpha} e_{\alpha}^{2} \bar{A}_{1 \alpha} \\
& =\frac{1}{8 \pi S_{T}^{i}} \int d \eta e^{-i \eta x} \sum_{\alpha} e_{\alpha}^{2}\langle P S| \bar{\psi}_{\alpha}\left(\xi^{-}\right)\left(\gamma^{i}-\frac{P^{i}}{P^{+}} \gamma^{+}\right) \gamma_{5} \psi_{\alpha}(0)+\text { h.c. }|P S\rangle .
\end{aligned}
$$

The above results are derived without recourse to perturbation theory, and also without the use of concept of collinear and massless partons. They are also the most general expressions for the leading contribution (in the $1 / q^{-}$expansion, not the leading contribution in terms of twists) to the deep inelastic structure functions in which the target is in an arbitrary Lorentz frame. Some of these expressions have not ever been obtained in previous works. The $x$-dependence of these structure functions is obvious in the above expressions. The scale $\left(Q^{2}\right)$-dependence is hidden in the hadronic bound states $|P S\rangle$ which can be described by multi-parton wave functions. In the next section, we shall analyze the complexities of structure functions in terms of bound states in our description.

\section{COMPLEXITIES OF STRUCTURE FUNCTIONS}

\section{A. Multi-parton wave functions}

As we have seen, the derivation of structure functions in the previous section is apparently quite different from the QCD improved (or field theoretical) parton model. The latter which is based on the collinear concept is purely an application of perturbation theory where further exploration of nonperturbative dynamics is lacking. Our description is also obviously very different from the OPE method. On the other hand, structure functions themselves are dominated by the nonperturbative quark-gluon dynamics. When we formulate them on the light-front, the structure functions are proportional to the simple hadronic matrix elements of the bilocal currents that are separated only in the longitudinal direction. In this formulation, no time evolution or propagation is explicitly involved in the matrix elements. Hence, unlike the OPE or the perturbative field theory descriptions of parton model, all the perturbative and nonperturbative dynamics here are completely carried by the structure of target's bound state. This is closer to the real physical picture probed in experiments.

The bound state of a hadron on light-front can be simply expanded in terms of the Fock states,

$$
|P S\rangle=\sum_{n, \lambda_{i}} \int^{\prime} d x_{i} d^{2} \kappa_{\perp i}\left|n, x_{i} P^{+}, x_{i} P_{\perp}+\kappa_{\perp i}, \lambda_{i}\right\rangle \Phi_{n}^{S}\left(x_{i}, \kappa_{\perp i}, \lambda_{i}\right)
$$


where $n$ represents $n$ constituents contained in the Fock state $\left|n, x_{i} P^{+}, x_{i} P_{\perp}+\kappa_{\perp i}, \lambda_{i}\right\rangle, \lambda_{i}$ is the helicity of the i-th constituent, $\int^{\prime}$ denotes the integral over the space:

$$
\sum_{i} x_{i}=1, \text { and } \sum_{i} \kappa_{\perp i}=0
$$

while $x_{i}$ is the fraction of the total longitudinal momentum carried by the $i$-th constituent, and $\kappa_{\perp i}$ is its relative transverse momentum with respect to the center mass frame:

$$
x_{i}=\frac{p_{i}^{+}}{P^{+}}, \quad \kappa_{i \perp}=p_{i \perp}-x_{i} P_{\perp}
$$

with $p_{i}^{+}, p_{i \perp}$ the longitudinal and transverse momenta of the $i$-th constituent. $\Phi_{n}^{S}\left(x_{i}, \kappa_{\perp i}, \lambda_{i}\right)$ is the amplitude of the Fock state $\left|n, x_{i} P^{+}, x_{i} P_{\perp}+\kappa_{\perp i}, \lambda_{i}\right\rangle$, i.e., the multi-parton wave function, which is boost invariant and satisfy the normalization condition:

$$
\sum_{n, \lambda_{i}} \int^{\prime} d x_{i} d^{2} \kappa_{\perp i}\left|\Phi_{n}^{S}\left(x_{i}, \kappa_{\perp i}, \lambda_{i}\right)\right|^{2}=1
$$

and is, in principle, determined from the light-front bound state equation,

$$
\left(M^{2}-\sum_{i=1}^{n} \frac{\kappa_{i \perp}^{2}+m_{i}^{2}}{x_{i}}\right)\left[\begin{array}{c}
\Phi_{q q q}^{S} \\
\Phi_{q q q g}^{S} \\
\vdots
\end{array}\right]=\left[\begin{array}{cc}
\left\langle q q q\left|H_{i n t}\right| q q q\right\rangle & \left\langle q q q\left|H_{i n t}\right| q q q g\right\rangle \\
\left\langle q q q g\left|H_{i n t}\right| q q q\right\rangle & \cdots \\
\vdots & \ddots
\end{array}\right]\left[\begin{array}{c}
\Phi_{q q q}^{S} \\
\Phi_{q q q g}^{S} \\
\vdots
\end{array}\right] .
$$

Here $H_{\text {int }}$ is the interaction part of the light-front QCD Hamiltonian [3]. Thus, the complexities of the structure functions carried by hadronic bound states are now translated into the language of multi-parton wave functions on the light-front, rather than composite operators in OPE.

Explicitly, let us look at the structure function $F_{2}\left(x, Q^{2}\right)$. It is found for the first time by us [12] that $F_{2}$ can be expressed in terms of a matrix element of either the plus component or the transverse component of the bilocal vector current. On the light-front, these two components have totally different operator structures but amazingly their matrix elements determine the same structure function.

The plus component (usually called the "good" component),

$$
\bar{\psi} \gamma^{+} \psi=2 \psi_{+}^{\dagger} \psi_{+}
$$

has no explicit dynamical dependence, and has the lowest mass dimension (a twist-two operator in OPE language). The corresponding matrix element has straightforward parton interpretation. It is clear from the above operator that on the light-front it is just a quark (parton) number operator which immediately leads to the fact that $F_{2}$ is proportional to parton density distributions $q_{\alpha}\left(x, Q^{2}\right)$.

$$
\begin{aligned}
\frac{F_{2}\left(x, Q^{2}\right)}{x} & =\sum_{\alpha} e_{\alpha}^{2} q_{\alpha}\left(x, Q^{2}\right) \\
q_{\alpha}\left(x, Q^{2}\right) & =\int d^{2} k_{\perp}\left\langle P S\left|\sum_{\lambda} b_{\alpha}^{\dagger}(k, \lambda) b_{\alpha}(k, \lambda)\right| P S\right\rangle \\
& =\int d^{2} \kappa_{\perp} \sum_{n, \lambda_{i}} \int^{\prime \prime} d x_{i} d^{2} \kappa_{\perp i}\left|\Phi_{n, \alpha}^{S}\left(x, x_{i}, \kappa_{\perp i}, \lambda_{i}\right)\right|^{2}
\end{aligned}
$$


where the $Q^{2}$-dependence is carried by the multi-parton wave functions with the active parton renormalized at the scale $Q^{2}, \int^{\prime \prime}$ denotes the integral in the right-hand-side over the space of Eq. (5.2) except for the active parton $\left(x, \kappa_{\perp}\right)=\left(k^{+} / P^{+}, p_{\perp}-x P_{\perp}\right)$. With this consideration it is straightforward to derive the logarithmic corrections that is the same as that obtained in the QCD improved parton model or in the OPE, as will be given in [1]. In this case, all the three descriptions are almost the same. The only difference here is that in our framework, the perturbative QCD dynamics is transferred from the composite operator into the scale-dependent multi-parton wave functions on the light-front, which enables us to describe the nonperturbative dynamics in the same framework.

But the transverse component (sometimes called the "bad" component),

$$
\bar{\psi} \gamma^{i} \psi=\bar{\psi}_{-} \gamma_{\perp}^{i} \psi_{+}+\bar{\psi}_{+} \gamma_{\perp}^{i} \psi_{-},
$$

depends explicitly on the fundamental quark-gluon interaction in QCD. According to the twist analysis [13], the transverse component of the bilocal current is a twist-three operator which has no simple parton interpretation. However, we have explicitly shown [12] that the corresponding matrix element of the above transverse component must have the same parton interpretation as that from the plus component. This is indeed obvious because they represent the same form factor of the bilocal current [see Eqs.(4.6) and (4.7)] and they describe the same structure function $F_{2}$ [see Eqs.4.12) and (4.13)]. This conclusion is very different from the current understanding of the twist of composite operators in the OPE or in the QCD improved parton model.

The explicit calculations in Ref. [12] further demonstrate that the real dynamics contained in the structure functions is determined by the matrix element with the rich information carried by the multi-parton wave functions. It is the complicated multi-parton wave functions that causes the same behavior for the matrix elements of the two apparently different operators (the plus and transverse components of bilocal current). However, this property was overlooked in previous works. Partly because one usually worked in a specific Lorentz frame, such as the rest frame or the infinite momentum frame in which the target's transverse momentum $P_{\perp}=0$, and also because enough attention was not paid on the hadronic bound state structure. With $P_{\perp}=0, F_{2}$ can only be expressed by Eq. (4.12). The expression in terms of the matrix element of the transverse component of the bilocal current, i.e. Eq. (4.13), does not exist if one lets $P_{\perp}=0$. In other words, picking up a specific Lorentz frame on light-front may lead to some ambiguities. In the next subsection, we shall present some discussion on such ambiguity in the concept of twist which is currently one of the most interesting topics in the study of deep inelastic processes.

\section{B. A Lorentz invariant definition of the twist}

As we have seen the lack of the additional expression (4.13) for $F_{2}$ in other approaches may be due to the specific choice of the Lorentz frame one often used. Indeed, since the deep inelastic processes are dominated by the physics close to the light-front, one often choose the following parameterization to analyze the structure functions [10,13:

$$
\begin{aligned}
P^{\mu} & =p^{\mu}+n^{\mu}, \\
q^{\mu} & =\frac{1}{M^{2}}\left(\nu-\sqrt{\nu^{2}+M^{2} Q^{2}}\right) p^{\mu}+\frac{1}{2}\left(\nu+\sqrt{\nu^{2}+M^{2} Q^{2}}\right) n^{\nu},
\end{aligned}
$$


where $p^{\mu}=(p, 0,0, p)$ and $n^{\mu}=(2,0,0,-2)$ are the light-like vectors; $p=M / 2$ yields the target rest frame and $p \rightarrow \infty$ leads to the infinite momentum frame. This choice of Lorentz frame for the deep inelastic processes may simplify the analysis, but it loses the generality when one tries to extract some general conclusions. A typical example is the twist analysis in the determination of the dominant contributions to deep inelastic structure functions.

The dominant contribution to the structure functions in terms of twist expansion is the basis of the OPE and QCD improved parton model analysis. Measuring the highertwist contributions has become a main topic in the current and future experiments since it may provide some nontrivial test of perturbative QCD beyond the logarithmic corrections. However, the definition of twist in literature is not unique and its physical interpretation is also not very transparent. According to standard definition, twist of a given composite operator is given by mass dimension of the operator minus its spin. In reality, this definition has no direct connection with experimental parameters.

Practically, the twist can be given in a less formal way: the twist of an invariant matrix element of a light-front bilocal operator, which controls the scaling behavior of the matrix element is determined by the power of $1 / Q$ that it contributes to deep inelastic processes [13. This definition is correct if the invariant matrix elements here are specified by those Lorentz invariant form factors of the bilocal currents given in Eqs. (4.6-4.10). However, in previous applications, one used the specific coordinates (5.10) to define the invariant matrix elements of the light-front bilocal currents and further used the usual dimensional analysis to determine the corresponding twists. Unfortunately, the twist of an matrix element defined in such specific coordinates with the usual dimensional analysis is not Lorentz invariant and contains ambiguous.

For example, according to the above definitions, Jaffe and Ji concluded that plus component of bilocal current is a twist-two operator, and transverse and minus components are twist three and four operators [13]. As a result, matrix elements of the transverse component of bilocal currents should be suppressed by a power of $1 / Q$ in comparison to the plus component matrix element. However, we find that the transverse component matrix element is actually suppressed by $P_{\perp}$ rather than $Q^{2}$ : Smaller the value $P_{\perp}$ takes in a specific choice of a Lorentz frame for the target, smaller will be the contribution arising from the transverse component matrix element. In the infinite momentum frame or in the target rest frame where the target has zero $P_{\perp}$, the transverse component matrix element must vanish. On the other hand, the plus component matrix element retains its value, no matter what the $Q^{2}$ value is (modulo logarithms). Thus, one cannot obtain the conclusion that transverse component of bilocal current is a twist-three operator that is suppressed by the factor $1 / Q$ in hard processes. The fact that the transverse component matrix element vanishes is a consequence of using the specific Lorentz frames with $P_{\perp}=0$ and is independent of the value of $Q^{2}$.

Indeed, we also find that twist of Lorentz invariant matrix elements of light-front bilocal currents can be properly defined in terms of light-front power counting analysis for light-cone dominated deep inelastic processes. Here one needs to consider the target in an arbitrary Lorentz frame. Then Eqs.(4.2)-(4.3) give the general definition of the Lorentz invariant matrix elements, i.e., the form factors of the bilocal vector and axial vector currents. The twist of a Lorentz invariant matrix element of bilocal currents is thus directly given by the mass dimension of the corresponding bilocal current form factors in light-front power 
counting. One must notice that light-front power counting is very different from the usual one [5]. The light-front power counting separately introduces different scales for transverse and longitudinal dimensions. Only the transverse dimension has mass scale,

$$
m: \frac{1}{x_{\perp}} .
$$

In light-front power counting [5], both the light-front quark field $\psi_{+}$and the gluon field $A_{\perp}$ have mass dimension one because their power assignments are given by

$$
\psi_{+}: \frac{1}{\sqrt{x^{-}}} \frac{1}{x_{\perp}}, \quad A_{\perp}: \frac{1}{x_{\perp}},
$$

although their longitudinal dimensions are different. Besides, the transverse derivative has mass dimension one and the longitudinal derivative has no mass dimension. Thus, from Eq. (3.9), we can see that $\psi_{-}: \frac{\sqrt{x^{-}}}{x_{\perp}^{2}}$ has mass dimension 2 .

In deep inelastic scattering processes, we have shown in Eqs.(4.2) and (4.3) that there are five Lorentz invariant form factors associated with the matrix elements of the bilocal vector and axial vector currents: $\bar{V}_{1 \alpha}, \bar{V}_{2 \alpha}, \bar{A}_{1 \alpha}, \bar{A}_{2 \alpha}$ and $\bar{A}_{3 \alpha}$. With simple power counting, one can easily find that three of them, $V_{1 \alpha}, \bar{A}_{1 \alpha}$ and $\bar{A}_{2 \alpha}$, are twist-two, while the remaining two, $\bar{A}_{2 \alpha}$ and $\bar{A}_{3 \alpha}$, are twist-four. Based on the normalization of bound state

$$
\left\langle P^{\prime} S^{\prime} \mid P S\right\rangle=2(2 \pi)^{3} P^{+} \delta^{3}\left(P-P^{\prime}\right) \delta_{S S^{\prime}}
$$

the light-front mass dimension of the state $|P S\rangle$ is -1 . Then we can easily count the mass dimension for each bilocal current form factor in light-front power counting as follows:

$$
\begin{aligned}
& \bar{V}_{1} \quad \sim\left\langle P S\left|\psi_{+}^{\dagger} \psi_{+}\right| P S\right\rangle \\
& \mathrm{m}-\operatorname{dim}\left(\bar{V}_{1}\right)=-1+1+1-1=0 \text {, } \\
& \text { or } \\
& \sim \frac{1}{P_{\perp}}\left\langle P S\left|\psi_{-}^{\dagger} \quad \psi_{+}\right| P S\right\rangle \\
& =\begin{array}{llllll}
-1 & -1 & +2 & +1 & -1 & =
\end{array} \text {, } \\
& \bar{A}_{1} \quad \sim \frac{1}{S_{T}}\left\langle P S\left|\psi_{-}^{\dagger} \psi_{+}\right| P S\right\rangle \\
& \mathrm{m}-\operatorname{dim}\left(\bar{A}_{1}\right)=\begin{array}{llllll}
-1 & -1 & +2 & +1 & -1 & =
\end{array} \text {, } \\
& \bar{A}_{2} \quad \sim \frac{1}{S_{T}}\left\langle P S\left|\psi_{-}^{\dagger} \psi_{+}\right| P S\right\rangle \\
& \mathrm{m}-\operatorname{dim}\left(\bar{A}_{2}\right)=\begin{array}{llllll}
-1 & -1 & +2 & +1 & -1 & =
\end{array} \text {, } \\
& \bar{V}_{2} \quad \sim\left\langle P S\left|\psi_{-}^{\dagger} \psi_{-}\right| P S\right\rangle \\
& \mathrm{m}-\operatorname{dim}\left(\bar{V}_{2}\right)=-1+2+2 \quad-1=2 \text {, } \\
& \bar{A}_{3} \quad \sim\left\langle P S\left|\psi_{-}^{\dagger} \psi_{-}\right| P S\right\rangle \\
& \mathrm{m}-\operatorname{dim}\left(\bar{A}_{3}\right)=-1+2+2 \quad-1=2 \text {. }
\end{aligned}
$$

The twist of the Lorentz invariant matrix elements (i.e. the bilocal current form factors) is defined as its mass dimension plus 2 . Therefore, $\bar{V}_{1 \alpha}, \bar{A}_{1 \alpha}$ and $\bar{A}_{2 \alpha}$ have twist-two, and $\bar{V}_{2 \alpha}$ and $\bar{A}_{3 \alpha}$ are twist-four Lorentz invariant matrix elements. 
As a matter of fact, there is indeed no twist-three Lorentz invariant matrix element in the leading contributions to deep-inelastic lepton-hadron processes. This conclusion should not be very surprising since experimentally no twist-three matrix element with a $1 / Q$ coefficient has been extracted in deep inelastic processes. Theoretically, it is also hard to find some result with power correction proportional to $1 / Q$ rather than $1 / Q^{2}$ in inclusive lepton-hadron deep inelastic processes. Our analysis shows that the next higher twist corrections to the leading twist-two contributions in deep inelastic processes are twist-four contributions which should be suppressed by the factor $1 / Q^{2}$. Indeed, from light-front power counting analysis, the mass dimension of Lorentz invariant bilocal current form factors is much more meaningful than the conventional definition of twist. If the light-front mass dimension of a form factor is zero, it means that there is no $1 / Q$ power suppression, see Eqs.(4.11), (4.15), and 4.17) for $F_{2}, g_{1}$ and $g_{T}$. If the mass dimension of a form factor is not zero, then there might be some $\mathcal{O}(1 / Q)$ power suppression to ensure that the structure function has the same dimensions [cf. Eq. (4.14) for $F_{L}$ ].

Now we can see that because the Lorentz invariant form factor $\bar{V}_{1 \alpha}$ is a twist-two matrix element, no matter if it is expressed in terms of the matrix element of plus or transverse component of the bilocal vector current, the leading contribution of $F_{2}$ is always a twist-two contribution, as is already known. The contribution of the transverse component matrix element goes to zero when we analyze it in the specific light-cone coordinates (5.10), but the ratio of the transverse component of the matrix element and $P_{\perp}$, which is proportional to $F_{2}$, remains invariant, as a result of Lorentz invariance. Thus the possible inconsistency of eqs.(4.12) and (4.13) in terms of the naive definition of twist does not really exist. It appears that the conventional definition of twist that is currently used in the literature may not be proper in the analysis of deep inelastic processes.

It is also to be noted that the twist analysis is meaningful only in some restricted portion of the phase space. For example, in the region of phase space connected to the limit $x \rightarrow 1$, that is when one of the particle in the Fock state is carrying almost all the hadron momenta, the states are far off its energy shell. Apparently, it seems that the contribution from such states to the structure functions are power suppressed, for it needs multiparton interaction in the first place to dump all the longitudinal momenta to one parton to produce such a Fock state. Yet their contributions are comparable to the leading twist ones where center of mass energy remains fixed. In fact, these contributions are more important and controls the behaviors of structure functions in the $x \rightarrow 1$ limit 15,16. In any case, as shown in these references, the twist expansion looses its significance all together in this limit.

Next, we shall analyze the twist of polarized structure functions, which has remained ambiguous for many years in the literature.

\section{Twist analysis of the polarized structure functions}

With the above analysis of the complexities of multi-parton wave functions and the new understanding of the concept of twist, we now explore the properties of the polarized structure functions $g_{1}$ and $g_{T}$.

In the literature, the properties of $g_{2}$ are usually discussed rather than the real transverse polarized structure function $g_{T}$ for historical reasons. But as we have pointed out, $g_{2}$ by itself has no real physical meaning. However, to clarify the confusion about the contributions 
of different twists to $g_{2}$ in the literature, we focus our attention on $g_{2}$ here. The polarized structure functions, especially the transverse polarized structure function, have recently received much theoretical and experimental attention. The polarized structure functions $g_{1}$ and $g_{2}$ are of great interest due to the fact that the former has been considered the best quantity to understand the origin of the proton spin, while the later may lead to the first observation of higher twist (twist-three) contribution in DIS. In the early naive parton model, Feynman claimed that $g_{2}$, just like $g_{1}$, has a simple parton interpretation because $g_{T}(x)=g_{1}(x)+g_{2}(x)=\frac{1}{2} \sum_{q} e_{q}^{2} \Delta q_{T}(x)$, where $\Delta q_{T}(x)$ is the distribution of transversely polarized quarks in a transversely polarized nucleon [8]. Wandzura and Wilczek studied the properties of $g_{2}$ in OPE analysis [17] and they claimed that except for a twist-three contribution $\bar{g}_{2}(x)$ which may be negligible in their model calculation, $g_{2}(x)$ can be related to an integral over $g_{1}(x)$ :

$$
g_{2}(x)=g_{2}^{W W}(x)+\bar{g}_{2}(x), \quad g_{2}^{W W}(x)=-g_{1}(x)+\int_{x}^{1} \frac{d y}{y} g_{1}(y) .
$$

The relation between $g_{2}^{W W}$ and $g_{1}(x)$ is called Wandzura-Wilczek (WW) relation. Later, Shuryak and Vainshtein pointed out that the twist-three contribution $\bar{g}_{2}$ is a direct quarkgluon interaction effect which is important for $g_{2}\left(x, Q^{2}\right)$ [18] so that the measurement of $g_{2}$ may also be very sensitive to the interaction dependent higher twist effects in QCD [13].

Here we shall apply light-front power counting to the analysis of the twist of polarized structure functions. The operator involved in $g_{1}$ is relatively simple, it is the plus component of bilocal axial vector current,

$$
\bar{\psi}_{\alpha}\left(\xi^{-}\right) \gamma^{+} \gamma_{5} \psi_{\alpha}=2 \psi_{+}^{\dagger}\left(\xi^{-}\right) \gamma_{5} \psi_{+}(0)
$$

which is a twist-two operator in the conventional definition. The corresponding Lorentz invariant matrix element is also twist-two in our definition. However, the operator determining the transverse polarized structure function $g_{2}$ is much more complicated. From Eqs. (4.16) and (4.18), one can obtain:

$$
\begin{gathered}
g_{2}\left(x, Q^{2}\right)=g_{T}\left(x, Q^{2}\right)-g_{1}\left(x, Q^{2}\right)=-\frac{1}{8 \pi} \int d \eta e^{-i \eta x} \sum_{\alpha} e_{\alpha}^{2} \frac{P^{+} \xi^{-}}{2} \bar{A}_{2 \alpha} \\
=\frac{1}{8 \pi S_{T}^{i}} \int d \eta e^{-i \eta x} \sum_{\alpha} e_{\alpha}^{2}\langle P S|\left[\bar{\psi}_{\alpha}\left(\xi^{-}\right)\left(\gamma^{i}-\frac{S^{i}}{S^{+}} \gamma^{+}\right) \gamma_{5} \psi_{\alpha}(0)\right. \\
+ \text { h.c. }]|P S\rangle .
\end{gathered}
$$

The operator in the above matrix element is a mixture of operators of the transverse and plus components of bilocal axial vector current and therefore involves twist-two and twistthree contributions in the conventional definition. On the light-front, it can be expressed as

$$
\bar{\psi}\left(\xi^{-}\right)\left(\gamma_{\perp}-\frac{S_{\perp}}{S^{+}} \gamma^{+}\right) \gamma_{5} \psi(0)=\frac{S^{i}}{S^{+}} O^{s}+O^{m}+O^{k_{\perp}}+O^{g}
$$

which corresponds to the conventional defining twist-two operator $O^{s}$ and twist-three operators of the quark mass part $O^{m}$, the quark transverse momentum part $O^{k_{\perp}}$ and the quark-gluon coupling part $O^{g}$, 


$$
\begin{aligned}
& O^{s}=-2 \psi_{+}^{\dagger}\left(\xi^{-}\right) \gamma_{5} \psi_{+}(0) \\
& O^{m}=m \psi_{+}^{\dagger}\left(\xi^{-}\right) \gamma_{\perp}\left(\frac{1}{i \overrightarrow{\partial^{+}}}-\frac{1}{i \overleftarrow{\partial}^{+}}\right) \gamma_{5} \psi_{+}(0) \\
& O^{k_{\perp}}=-\psi_{+}^{\dagger}\left(\xi^{-}\right)\left(\gamma_{\perp} \frac{1}{\vec{\partial}^{+}} \vec{\partial}_{\perp}+\overleftarrow{\not_{\perp}} \frac{1}{\check{\partial}^{+}} \gamma_{\perp}\right) \gamma_{5} \psi_{+}(0) \\
& O^{g}=g \psi_{+}^{\dagger}\left(\xi^{-}\right)\left(\not A_{\perp}\left(\xi^{-}\right) \frac{1}{i \overleftarrow{\partial}^{+}} \gamma_{\perp}-\gamma_{\perp} \frac{1}{i \vec{\partial}^{+}} \not A_{\perp}(0)\right) \gamma_{5} \psi_{+}(0)
\end{aligned}
$$

where $m$ and $g$ are the quark mass and quark-gluon coupling constant in QCD, and $A_{\perp}=$ $A_{\perp}^{a} T_{a}$ is the transverse gauge field. The main concern in the previous works is which operator dominates $g_{2}$ in hard processes in terms of twist analysis.

However, Eqs. (5.16) and (4.16) show that $g_{T}$ is determined by Lorentz invariant form factor of the bilocal axial vector current, $\bar{A}_{1 \alpha}$, while $g_{1}$ is determined by both $\bar{A}_{2 \alpha}$ and $\bar{A}_{1 \alpha}$. According to our analysis on twist, both $\bar{A}_{1 \alpha}$ and $\bar{A}_{2 \alpha}$ are twist-two invariant matrix elements because their mass dimensions are zero. Although these operators are apparently so complicated, their contributions (in terms of matrix elements) to $g_{2}$ are indeed twist-two regardless if they depend on the quark mass, quark transverse momentum and the direct quark-gluon coupling. As a matter of fact, it is not meaningful to separate $g_{2}$ into twist-two and twist-three parts as in Eq.(5.14). In our recent work on the validity of Wandzura-Wilczek relation, we have shown that such a separation is strongly violated in perturbative QCD. The violation originates from the cancellation between the naive twist-two and twist-three contributions due to the multi-parton structure of wave functions [19]. Such a cancellation is hardly understood in the conventional twist expansion if one believes that the higher twist is suppressed by an order of $1 / Q$ in comparison with the lower twist. Now, we understand that there would be no such ambiguity in the twist analysis contributions of $g_{2}$, if one used the definition of twist based on light-front power counting and the Lorentz invariant matrix elements of the bilocal currents (rather than the bilocal current operators themselves).

In conclusion, the complexities of deep inelastic structure functions are mainly carried by the multi-parton wave functions of the hadrons which completely determine the Lorentz invaraint matrix element (i.e. the form factors) of the bilocal currents.

\section{A scheme for the evaluation of soft and hard contributions to deep inelastic structure functions}

Up to this point, all the derivations and discussions of the deep inelastic structure functions in the $1 / q^{-}$expansion are rigorously carried out within light-front QCD and without recourse to perturbation theory. The remaining problem is how to evaluate various matrix elements of bilocal currents. These matrix elements contain both hard and soft quark and gluon dynamics. As we have analyzed in this section, all the hard and soft dynamics probed through the structure functions are completely carried by the target's bound state in the present formulation. This is the main advantage of this formalism that allows us to explore the perturbative and nonperturbative contributions to the structure functions in the same framework. In the rest of this section, we shall propose a scheme for such an exploration.

In Sec. V.A, the hadronic bound state is formally expressed in terms of Fock space expansion on the light-front by Eq. (5.1), and it is determined in principle by the light-front 
bound state equation of Eq. (5.5). However, the difficulty in determining wave functions by solving Eq. (5.5) is that the QCD Hamiltonian contains more than one energy scale. At different energy scales, QCD Hamiltonian can exhibit different aspects of the dynamics. Let us roughly divide the quark and gluon dynamics into two energy domains, namely, high energy and low energy. In the high energy domain, the dynamics is controlled by the renormalized QCD Hamiltonian with all the constituents carrying momenta greater than a scale $\mu_{\text {fact }}(\approx 1 G e V)$ which we call the factorization energy scale. This high energy QCD Hamiltonian describes all the hard dynamics of quarks and gluons and determines the hard contributions to the structure functions which can be calculated in the perturbation theory. In the low energy domain, the effective QCD Hamiltonian is still unknown but such a low energy QCD Hamiltonian should fairly determine the low energy structure of the hadrons and is responsible for the soft contributions to the structure functions.

Schematically, we may write the QCD Hamiltonian on the light-front for DIS as

$$
H_{Q C D}^{L F}= \begin{cases}H_{Q C D}^{H} \equiv \int_{k_{i \perp}^{2} \geq \mu_{\text {fact }}^{2}} d k_{i}^{+} d^{2} k_{i \perp} \mathcal{H}_{Q C D}^{C}\left(k_{i}\right) & \text { for hard contributions } \\ H_{Q C D}^{M} \equiv \int d k_{i}^{+} d^{2} k_{i \perp} \mathcal{H}_{Q C D}^{C}\left(k_{i}\right) & \text { for mixed hard and soft modes } \\ H_{Q C D}^{L} \equiv \int_{k_{i \perp}^{2}<\mu_{\text {fact }}^{2}} d k_{i}^{+} d^{2} k_{i \perp} \mathcal{H}_{Q C D}^{L}\left(k_{i}\right) & \text { for soft contributions }\end{cases}
$$

where $H_{Q C D}^{H}$ represents the canonical light-front QCD Hamiltonian (with density $\mathcal{H}_{Q C D}^{C}$ given in [3]) in which the transverse momenta of all the quarks and gluons are restricted to be $\mu_{\text {fact }}^{2}<k_{\perp}^{2}<Q^{2}$ (i.e., hard partons), and $H_{Q C D}^{L}$ denotes a low energy effective light-front Hamiltonian in which all the constituents have the transverse momentum $k_{\perp}^{2}<\mu_{\text {fact }}^{2}$ (soft partons). This low energy Hamiltonian is, in principle, obtained by integrating out all modes with $k_{\perp}^{2}>\mu_{\text {fact }}^{2}$ from the canonical light-front QCD Hamiltonian, which leads to $\mathcal{H}_{Q C D}^{L}$. In addition, we also introduce a Hamiltonian $H_{Q C D}^{M}$ which depends only on the interaction part and which mixes the hard and soft partons. Writing the light-front QCD Hamiltonian in such three parts will make the discussion of the perturbative and nonperturbative QCD contributions to DIS structure functions much more transparent, as we will see next.

Now, the target bound state can be expressed by

$$
|P S\rangle=U_{h}\left|P S, \mu_{\text {fact }}^{2}\right\rangle,
$$

with

$$
\begin{aligned}
& U_{h}=T^{+} \exp \left\{-\frac{i}{2} \int_{-\infty}^{0} d x^{+}\left(H_{Q C D}^{H}+H_{Q C D}^{M}\right)\right\}, \\
& H_{Q C D}^{L}\left|P S, \mu_{\text {fact }}^{2}\right\rangle=\frac{P_{\perp}^{2}+M^{2}}{P^{+}}\left|P S, \mu_{\text {fact }}^{2}\right\rangle .
\end{aligned}
$$

In Eq. (5.21), $H^{H}$ and $H^{M}$ contain the interaction parts only and the mixed Hamiltonian $H_{Q C D}^{M}$ is active only in the extreme right of the time-ordered expansion. In other words, the hard and the soft dynamics in the bound states are determined separately by $H_{Q C D}^{H}$ and $H_{Q C D}^{L}$ but these two contributions are connected by $H_{Q C D}^{M}$ through the time-ordered 
expansion of Eq. (5.21) on the state $\left|P S, \mu_{\text {fact }}^{2}\right\rangle$ in Eq. (5.20), where the soft dynamics, represented by $\left|P S, \mu_{\text {fact }}^{2}\right\rangle$, must be solved nonperturbatively from Eq. (5.22), and the key point to solve Eq. (5.22) is to find the low energy effective Hamiltonian $H_{Q C D}^{L}$. A practical procedure to find $H_{Q C D}^{L}$ on the light-front may be the use of similarity renormalization group approach plus a weak-coupling treatment developed recently [5-7]. Indeed, a major effort on the study of light-front QCD is underway at present to solve this problem [26].

To see how the perturbative and nonperturbative QCD contributions can be separately evaluated in the present formalism and how these two contributions are connected by $H_{Q C D}^{M}$, we substitute Eqs. (5.20 5.22) into the expressions of structure functions. Denote the structure functions simply by $F_{i} \equiv:\left\{F_{L}, F_{2}, g_{1}, g_{T}\right\}$,

$$
F_{i}\left(x, Q^{2}\right) \sim \int d \eta e^{-i \eta x} \sum_{\alpha} e_{\alpha}^{2}\left\langle P S\left|\bar{\psi}_{\alpha}\left(\xi^{-}\right) \Gamma_{i} \psi_{\alpha}(0) \pm h . c .\right| P S\right\rangle
$$

where $\Gamma_{i}$ involves the Dirac $\gamma$-matrices [see Eqs. (4.12)-(4.18)]. It follows that

$$
\begin{aligned}
F_{i}\left(x, Q^{2}\right)=\int d \eta e^{-i \eta x} & \sum_{\alpha} e_{\alpha}^{2} \sum_{n_{1}, n_{2}}\left\langle P S, \mu_{\text {fact }}^{2} \mid n_{1}\right\rangle\left\langle n_{2} \mid P S, \mu_{\text {fact }}^{2}\right\rangle \\
& \times\left\langle n_{1}\left|U_{h}^{-1}\left[\bar{\psi}_{\alpha}\left(\xi^{-}\right) \Gamma_{i} \psi_{\alpha}(0) \pm h . c\right] U_{h}\right| n_{2}\right\rangle,
\end{aligned}
$$

where $\left|n_{1}\right\rangle,\left|n_{2}\right\rangle$ are a complete set of quark and gluon Fock states with momentum $k_{i}^{2} \leq$ $\mu_{\text {fact }}^{2}$. This is indeed the generalized factorization theorem in the light-front Hamiltonian formulation. The hard contribution is described by the matrix element,

$$
\left\langle n_{1}\left|U_{h}^{-1}\left[\bar{\psi}_{\alpha}\left(\xi^{-}\right) \Gamma_{i} \psi_{\alpha}(0) \pm h . c\right] U_{h}\right| n_{2}\right\rangle,
$$

which can be evaluated in the light-front time-ordered perturbation theory [3]. The physical picture corresponds to the multi-parton forward scattering amplitude with all the internal partons carrying a momentum with the transverse component $k_{\perp}: \mu_{\text {fact }}^{2} \leq k_{\perp}^{2} \leq Q^{2}$ and the longitudinal momentum fraction $y: x \leq y \leq 1$. The soft contribution is characterized by the overlap of the multi-parton wave functions in different Fock states:

$$
\left\langle P S, \mu_{\text {fact }}^{2} \mid n_{1}\right\rangle\left\langle n_{2} \mid P S, \mu_{\text {fact }}^{2}\right\rangle,
$$

which contains all the quantum correlations and interference effects of multi-parton (quarks and gluons) dynamics in the low energy domain with $k_{\perp}^{2}<\mu_{\text {fact }}^{2}$. Since all the internal partons in the time-ordered expansion of $U_{h}$ in Eq. (5.25) carry momenta $\mu_{\text {fact }}^{2} \leq k_{\perp}^{2} \leq Q^{2}$, the mixed Hamiltonian $H_{Q C D}^{M}$ has the contribution only in the extreme left and extreme right of the time-ordered products. It is this effect that connects the hard contribution of Eq. (5.25) to the soft contribution Eq. (5.26). We will present more detailed discussion in [1].

The simple parton picture in deep inelastic processes corresponds to the case of $\left|n_{1}\right\rangle=$ $\left|n_{2}\right\rangle$ in Eq. (5.24) with only one parton in $\left|n_{1}\right\rangle$ actively participating in the high energy process, all others being spectators. This immediately leads to

$$
F_{i}\left(x, Q^{2}\right) \sim \sum_{\alpha} e_{\alpha}^{2} \int_{x}^{1} d y \mathcal{P}_{p p^{\prime}, i}\left(y, x, \frac{Q^{2}}{\mu_{\text {fact }}^{2}}\right) q_{\alpha i}\left(y, \mu_{\text {fact }}^{2}\right),
$$


where the hard scattering coefficient $\mathcal{P}_{p p^{\prime}, i}$ is determined by

$$
\mathcal{P}_{p p^{\prime}, i}\left(y, x, \frac{Q^{2}}{\mu_{\text {fact }}^{2}}\right) \simeq \int d \eta e^{-i \eta x}\left\langle y, k_{\perp}, s\left|U_{h}^{-1}\left[\bar{\psi}_{\alpha}\left(\xi^{-}\right) \Gamma_{i} \psi_{\alpha}(0) \mp h . c .\right] U_{h}\right| y, k_{\perp}, s\right\rangle .
$$

Here we have denoted $\left|y, k_{\perp}, s\right\rangle\left(y=k^{+} / P^{+}\right)$as the active parton state. Eq. (5.28) means that we have suppressed all references to the spectators in the states $\left|n_{1}\right\rangle$. The hard scattering coefficient is directly related to the so-called the splitting function whose physical interpretation is the probability to find a daughter parton $p^{\prime}$ in the active parent parton $p$. The quantity $q_{\alpha i}\left(y, \mu_{\text {fact }}^{2}\right)$, usually called the parton distribution function, is given by

$$
q_{\alpha i}\left(y, \mu_{\text {fact }}^{2}\right)=\sum_{n}\left|\left\langle P S, \mu_{\text {fact }}^{2} \mid n\right\rangle\right|^{2},
$$

where $n$ runs over all the Fock states containing the active parton with momentum fraction $y$. Theoretically, the parton distributions are determined by solving Eq. (5.22). Physically, they contain only the quantum correlations of multi-parton dynamics but no quantum interference effects. Example of such distribution functions is given by Eqs. (5.75.8) for $F_{2}(x) / x$ which manifestly exhibits the simple parton picture. For detailed calculations also see Ref. [1].

The above discussions indeed constitute a presentation of factorization scheme in the light-front Hamiltonian formulation. The leading hard contributions to the structure func-

tions are given by the the hard scattering coefficient $\mathcal{P}_{p p^{\prime}, i}\left(y, x, \frac{Q^{2}}{\mu_{\text {fact }}^{2}}\right)$ and a complete calculation of $\mathcal{P}_{p p^{\prime}, i}$ based on the light-front time-ordered perturbative expansion of the multiparton wave functions will be presented in a subsequent paper [1]. The evaluation of soft contribution to the structure functions, given by $q_{\alpha i}\left(x, \mu_{\text {fact }}^{2}\right)$ remains for future investigations of nonperturbative light-front QCD approaches to the hadronic bound states. Other higher order contributions can also be systematically evaluated from Eqs. (5.25) and (5.26) [1]. Thus, a unified treatment of both perturbative and nonperturbative aspects of deep inelastic structure functions in the same framework may emerge which permits one to overcome the obstacles in dealing with the nonperturbative QCD dynamics in OPE and field theoretical parton model approaches.

\section{PHYSICAL INTERPRETATION OF THE STRUCTURE FUNCTIONS FROM SUM RULES}

In this last section, we shall explore the physical meaning of the deep inelastic structure functions in our framework of light-front QCD. The physical meaning of the structure functions can be easily understood from the sum rules they obey. Some of them have been known for long time but others are new. Sum rules generally arise from the existence of conservation laws. First we consider the case of sum rules in unpolarized deep inelastic scattering for which a detailed consideration of the energy-momentum density in QCD is necessary.

\section{A. Energy-momentum tensor in QCD}

The symmetric, gauge-invariant energy-momentum tensor in QCD is given by 


$$
\begin{aligned}
\theta^{\mu \nu}= & \frac{1}{2} \bar{\psi} i\left[\gamma^{\mu} D^{\nu}+\gamma^{\nu} D^{\mu}\right] \psi-F^{\mu \lambda a} F_{\lambda a}^{\nu}+\frac{1}{4} g^{\mu \nu}\left(F_{\lambda \sigma a}\right)^{2} \\
& -g^{\mu \nu} \bar{\psi}\left(i \gamma^{\lambda} D_{\lambda}-m\right) \psi .
\end{aligned}
$$

The last term vanishes using the equation of motion. Formally, we split the energy momentum tensor into a "fermionic" part $\theta_{q}^{\mu \nu}$ and a "gauge bosonic" part $\theta_{g}^{\mu \nu}$ :

$$
\theta_{q}^{\mu \nu}=\frac{1}{2} \bar{\psi} i\left[\gamma^{\mu} D^{\nu}+\gamma^{\nu} D^{\mu}\right] \psi
$$

and

$$
\theta_{g}^{\mu \nu}=-F^{\mu \lambda a} F_{\lambda a}^{\nu}+\frac{1}{4} g^{\mu \nu}\left(F_{\lambda \sigma a}\right)^{2},
$$

with $F_{\lambda a}^{\nu}=\partial^{\nu} A_{\lambda a}-\partial_{\lambda} A_{a}^{\nu}+g f_{a b c} A_{b}^{\nu} A_{\lambda c}$. To be consistent with the study of deep inelastic structure function which is formulated in $A^{+}=0$ gauge, we shall work in the same gauge.

We have, for the fermionic part of the longitudinal momentum density,

$$
\begin{gathered}
\theta_{q}^{++}=i \bar{\psi} \gamma^{+} \partial^{+} \psi \\
\theta_{g}^{++}=-F_{\lambda}^{+\lambda} F_{\lambda}^{+}=\partial^{+} A^{i} \partial^{+} A^{i} .
\end{gathered}
$$

Thus

$$
\theta^{++}=i \bar{\psi} \gamma^{+} \partial^{+} \psi+\partial^{+} A^{i} \partial^{+} A^{i}
$$

free of interactions at the operator level itself. The longitudinal momentum operator

$$
P^{+}=\frac{1}{2} \int d x^{-} d^{2} x_{\perp} \theta^{++}
$$

Next consider the transverse momentum density

$$
\theta_{q}^{+i}=\frac{1}{2} \bar{\psi} i\left[\gamma^{+} D^{i}+\gamma^{i} D^{+}\right] \psi=\theta_{q-1}^{+i}+\theta_{q-2}^{+i}
$$

with

$$
\theta_{q-1}^{+i}=\frac{1}{2} \bar{\psi} i \gamma^{+} D^{i} \psi \text { and } \theta_{q-2}^{+i}=\frac{1}{2} \bar{\psi} i \gamma^{i} \partial^{+} \psi
$$

For the Hamiltonian density, the fermionic part is given by

$$
\theta_{q}^{+-}=\theta_{q}^{+-(1)}+\theta_{q}^{+-(2)}
$$

with

$$
\theta_{q}^{+-(1)}=i \psi^{+\dagger} \partial^{-} \psi^{+}+g \psi^{+\dagger} A^{-} \psi^{+},
$$

and 


$$
\theta_{q}^{+-(2)}=i \psi^{-\dagger} \partial^{+} \psi^{-}
$$

Using the Dirac equation for the fermion, we find that $\theta_{q}^{+-(1)}=\theta_{q}^{+-(2)}$. Thus we have,

$$
\begin{aligned}
\theta_{q}^{+-} & =i \bar{\psi} \gamma^{-} \partial^{+} \psi=2 i \psi^{-\dagger} \partial^{+} \psi^{-} \\
& =2 \psi^{+\dagger}\left[\alpha_{\perp} \cdot\left(i \partial_{\perp}+g A_{\perp}\right)+\gamma^{0} m\right] \frac{1}{i \partial^{+}}\left[\alpha_{\perp} \cdot\left(i \partial_{\perp}+g A_{\perp}\right)+\gamma^{0} m\right] \psi^{+}
\end{aligned}
$$

The gauge boson part of the Hamiltonian density is more complicated [3]:

$$
\begin{aligned}
\theta_{g}^{+-}=-F^{+\lambda a} F_{\lambda a}^{-}+\frac{1}{4} g^{+-}\left(F_{\lambda \sigma a}\right)^{2}=\frac{1}{4}\left(\partial^{+} A^{-a}\right)^{2}+\frac{1}{2} F^{i j a} F_{i j}^{a} \\
=\left(\partial^{i} A_{a}^{j}\right)^{2}+2 g f^{a b c} A_{a}^{i} A_{b}^{j} \partial^{i} A_{c}^{j}+\frac{g^{2}}{2} f^{a b c} f^{a d e} A_{b}^{i} A_{c}^{j} A_{d}^{i} A_{e}^{j} \\
+2 g \partial^{i} A_{a}^{i}\left(\frac{1}{\partial^{+}}\right)\left(f^{a b c} A_{b}^{j} \partial^{+} A_{c}^{j}+2\left(\psi^{+}\right)^{\dagger} T^{a} \psi^{+}\right) \\
+g^{2}\left(\frac{1}{\partial^{+}}\right)\left(f^{a b c} A_{b}^{i} \partial^{+} A_{c}^{i}+2\left(\psi^{+}\right)^{\dagger} T^{a} \psi^{+}\right)\left(\frac{1}{\partial^{+}}\right)\left(f^{a d e} A_{d}^{j} \partial^{+} A_{e}^{j}+2\left(\psi^{+}\right)^{\dagger} T^{a} \psi^{+}\right)
\end{aligned}
$$

where we have used the equation of constraint for the gauge field.

Next, we discuss the physical interpretation of the deep inelastic structure functions on the basis of the sum rules they obey.

\section{B. Longitudinal momentum sum rule}

The content of the momentum sum rule is known for long time. For completeness, we shall rederive it in our framework. The sum rule is simply that if we add up the longitudinal momentum fractions carried by all the quarks, antiquarks, and the gluons (alternatively by the valence quarks, sea quarks and the gluons) in the nucleon we should get one. ¿From the expression of $F_{2}$ in terms of the plus component of the bilocal current matrix element given in Eq.(4.12) we have

$$
\int_{0}^{1} d x F_{2}(x)=\left(\frac{1}{2\left(P^{+}\right)^{2}}\right) \sum_{\alpha} e_{\alpha}^{2}\left\langle P\left|\theta_{F \alpha}^{++}\right| P\right\rangle .
$$

Formally, we can define the "gluon structure function" 10

$$
F_{2}^{G}(x)=\frac{1}{4 \pi P^{+}} \int d \eta e^{-i \eta x}\left\langle P\left|F^{+\nu a}\left(\xi^{-}\right) F_{\nu}^{+a}(0)\right| P\right\rangle
$$

so that,

$$
\int_{0}^{1} d x F_{2}^{G}(x)=\left(\frac{1}{2\left(P^{+}\right)^{2}}\right)\left\langle P\left|\theta_{G}^{++}\right| P\right\rangle .
$$

Only if we assume $e_{\alpha}=1$, one can obtain the momentum sum rule 


$$
\int_{0}^{1} d x\left[F_{2}+F_{2}^{G}\right]=1
$$

Similarly, from Eq.(4.13) in terms of the transverse component of bilocal current matrix element, we have

$$
\begin{aligned}
\int d x F_{2}\left(x, Q^{2}\right) & =\frac{1}{2 P^{+} P_{\perp}^{i}} \sum_{\alpha} e_{\alpha}^{2}\left\langle P S\left|\bar{\psi}(0) \gamma^{+}\left(i \stackrel{\leftrightarrow}{\partial^{i}}\right) \psi_{\alpha}(0)\right| P S\right\rangle \\
& =\frac{1}{P^{+} P_{\perp}^{i}} \sum_{\alpha} e_{\alpha}^{2}\left\langle P S\left|\theta_{F \alpha}^{+i}\right| P S\right\rangle
\end{aligned}
$$

and the momentum sum rule can also be written as

$$
\frac{1}{2 P^{+} P_{\perp}^{i}}\left\langle P\left|\theta^{+i}\right| P\right\rangle=\frac{1}{2 P^{+} P_{\perp}^{i}}\left\langle P\left|\left[\theta_{F}^{+i}+\theta_{G}^{+i}\right]\right| P\right\rangle=1 .
$$

The sum rule given in Eq.(6.16) means that $F_{2}$ measures the longitudinal momentum distribution of quarks inside the hadrons, as known long time ago. From Eqs.(6.16) and (6.20) we observe that the hadron expectation value of the longitudinal and transverse momentum densities gives the same information, namely, the total longitudinal momentum fraction carried by the partons.

We note here an apparent paradox that results when one ignores the essential complexities carried by the state. The operators corresponding to the transverse momentum density explicitly depend on the interaction since $D^{i}=\partial^{i}-i g A^{i}$ and $\theta_{q-2}^{+i}$ depends on $\psi^{-}$which in turn depends explicitly on the interaction. Since we know that $P^{i}$ is a kinematical operator this appears puzzling at first sight. Thus we expect the apparent dependence of $P^{i}$ on the interaction to be spurious. However this cannot be demonstrated at the level of operators alone. But this is not a serious problem since what really matters are the matrix elements.

Indeed, our demonstration [12 that the matrix element of the transverse component of the vector bilocal has the same parton interpretation as that of the plus component and hence the apparent interaction dependence in the former is completely spurious in turns directly tells us that the interaction dependence of the operator $\theta_{q-2}^{+i}$ is completely spurious. In that case an explicit evaluation of off-diagonal matrix elements in the Fock space expansion of states is involved. Similarly, an explicit demonstration shows that $\theta_{q-1}^{+i}$ has no interaction dependence at the level of matrix element, namely

$$
\theta_{q-1}^{+i}=\frac{1}{2} i \bar{\psi} i \gamma^{+} \partial^{i} \psi+g\left(\psi^{+}\right)^{\dagger} A^{i} \psi^{+}
$$

but the matrix elements of the second term vanishes. Then, at the level of matrix elements

$$
\theta_{q}^{+i}=i \bar{\psi} \gamma^{i} \partial^{+} \psi
$$

This demonstration clearly shows that drawing conclusions by looking at the operator structure is quite misleading in the case of operators that are twist three in the conventional definition.

Since $F_{2}$ involves quark charges in specific combinations, it does not give the direct test of the above momentum sum rule. To test the sum rule experimentally, one can combine 
the data for both the electron-proton and electron-neutron deep-inelastic scatterings and assume that the sea is flavor symmetric, then

$$
\begin{aligned}
\int d x\left[F_{2}^{e p}(x)+F_{2}^{e n}(x)\right] & =\frac{5}{9} \frac{1}{\left(P^{+}\right)^{2}} \sum_{\alpha}\left\langle P S\left|\theta_{F \alpha}^{++}\right| P S\right\rangle \\
& =\frac{5}{9} \frac{1}{P^{+} P_{\perp}^{i}} \sum_{\alpha}\left\langle P S\left|\theta_{F \alpha}^{+i}\right| P S\right\rangle .
\end{aligned}
$$

This shows that $\frac{9}{5} \int d x\left[F_{2}^{e p}(x)+F_{2}^{e n}(x)\right]$ is the total longitudinal momentum fraction carried by the all the quarks in proton and neutron. If the quarks carry all the momentum, then we expect that

$$
\int d x\left[F_{2}^{e p}(x)+F_{2}^{e n}(x)\right]=\frac{5}{9} .
$$

Experimental data shows that the above integral is 0.28 . In other words, as is well-known, there are half of the momentum in hadrons are carried by gluons or the see quarks if the sea is not flavor symmetric.

\section{Sum rule for $g_{1}$}

Now we consider the sum rule for $g_{1}$ and its physical interpretation. Integrating $g_{1}$ over $x$, we simply have

$$
\Gamma_{1}\left(Q^{2}\right)=\int d x g_{1}\left(x, Q^{2}\right)=\frac{1}{2 S^{+}} \sum_{\alpha} e_{\alpha}^{2}\left\langle P S\left|\bar{\psi}_{\alpha}(0) \gamma^{+} \gamma_{5} \psi_{\alpha}(0)\right| P S\right\rangle
$$

Note that

$$
\left\langle P S\left|\bar{\psi}_{\alpha}(0) \gamma^{+} \gamma_{5} \psi_{\alpha}(0)\right| P S\right\rangle=\Delta q_{\alpha}^{G I}\left(Q^{2}\right) S^{+}
$$

where $\Delta q_{\alpha}^{G I}$ is the distribution function of chirality carried by all the quarks in the longitudinally polarized target and renormalized in the gauge invariant scheme. The above form can also be directly obtained from Eq. (4.3). Then, we have

$$
\Gamma_{1}\left(Q^{2}\right)=\frac{1}{2} \sum_{\alpha} e_{\alpha}^{2} \Delta q_{\alpha}^{G I}\left(Q^{2}\right)
$$

If one uses the chiral invariant renormalization scheme, the first moment of $g_{1}$ also exhibits the anomaly contribution [21]. We will discuss how this property manifests in the light-front Hamiltonian formulation in a separate publication.

It is clear now that $g_{1}$ describes the distribution of chirality carried by the quarks inside the target (proton or neutron). Note the first moment $\Gamma_{1}$ is usually called the proton's spin structure function. But one must also be aware that on the light-front, the plus component of axial current is the same as the third component of the quark helicity operator density on the light-front. Therefore, its expectation value is the same as the third component of the spin on the light-front (i.e., light-front helicity), which is not the same as the $z$-component of intrinsic spin defined in the rest frame of the equal-time coordinates. On the light-front, 
as is well-known, there is a very complicated relation between the light-front helicity and the intrinsic spin in the rest frame. This relation depends on the interactions in the fundamental theory. At present, one only knows the exact relation for free theory [22]. In other words,

$g_{1}$ does not really measure the spin of proton. Simply calling Eq. (6.28) a spin sum rule is misleading.

\section{Light-front helicity sum rule}

For the fermions, the intrinsic light-front helicity distribution function is given by

$$
\Delta q\left(x, Q^{2}\right)=\frac{1}{8 \pi S^{+}} \int d \eta e^{-i \eta x}\left\langle P S\left|\left[\bar{\psi}\left(\xi^{-}\right) \gamma^{+} \Sigma^{3} \psi(0)+h . c\right]\right| P S\right\rangle
$$

where $\Sigma^{3}=i \gamma^{1} \gamma^{2}$. This is the same as the chirality distribution function $g_{1}$.

We define the orbital helicity distribution for the fermion

$$
\Delta q_{L}\left(x, Q^{2}\right)=\frac{1}{4 \pi P^{+}} \int d \eta e^{-i \eta x}\langle P S|\left[\bar{\psi}\left(\xi^{-}\right) \gamma^{+} i\left(x^{1} \partial^{2}-x^{2} \partial^{1}\right) \psi(0)+\text { h.c. }\right]|P S\rangle .
$$

For the gluon, the intrinsic light-front helicity distribution is defined [23] as

$$
\Delta g\left(x, Q^{2}\right)=-\frac{i}{4 \pi\left(P^{+}\right)^{2} x} \int d \eta e^{-i \eta x}\left\langle P S\left|F^{+\alpha}\left(\xi^{-}\right) \tilde{F}_{\alpha(0)}^{+}\right| P S\right\rangle .
$$

The dual tensor

$$
\tilde{F}^{\mu \nu}=\frac{1}{2} \epsilon^{\mu \nu \rho \sigma} F_{\rho \sigma} \quad \text { with } \quad \epsilon^{+1-2}=2 .
$$

We also define the light-front orbital helicity distribution for the gluon as

$$
\begin{aligned}
\Delta g_{L}\left(x, Q^{2}\right)=-\frac{1}{4 \pi P^{+}} \int d \eta e^{-i \eta x} & \langle P S|\left[x^{1} F^{+\alpha}\left(\xi^{-}\right) \partial^{2} A_{\alpha}(0)\right. \\
& \left.-x^{2} F^{+\alpha}\left(\xi^{-}\right) \partial^{1} A_{\alpha}(0)\right]|P S\rangle .
\end{aligned}
$$

Note that all the above distribution functions are defined in the light-front gauge $A^{+}=0$.

The light-front helicity operator is given by

$$
J^{3}=\frac{1}{2} \int d x^{-} d^{2} x^{\perp}\left[x^{1} \theta^{+2}-x^{2} \theta^{+1}\right]
$$

where $\theta^{\mu \nu}$ is the symmetric energy momentum tensor. Explicitly, the fermion orbital helicity operator

$$
J_{q(o)}^{3}=i \int d x^{-} d^{2} x^{\perp} \psi^{+\dagger}\left[x^{1} \partial^{2}-x^{2} \partial^{1}\right] \psi^{+}
$$

and the fermion intrinsic helicity operator

$$
J_{q(i)}^{3}=\frac{1}{2} \int d x^{-} d^{2} x^{\perp} \psi^{+\dagger} \Sigma^{3} \psi^{+} .
$$


The gluon orbital helicity operator

$$
J_{g(o)}^{3}=\frac{1}{2} \int d x^{-} d^{2} x^{\perp}\left[x^{1}\left(\partial^{+} A^{1} \partial^{2} A^{1}+\partial^{+} A^{2} \partial^{2} A^{2}\right)-x^{2}\left(\partial^{+} A^{1} \partial^{1} A^{1}+\partial^{+} A^{2} \partial^{1} A^{2}\right)\right]
$$

and the gluon intrinsic helicity operator

$$
J_{g(i)}^{3}=\frac{1}{2} \int d x^{-} d^{2} x^{\perp}\left[A^{1} \partial^{+} A^{2}-A^{2} \partial^{+} A^{1}\right] .
$$

The helicity sum rule for the nucleon target implies

$$
\frac{1}{\mathcal{N}}\left\langle P S\left|\left[J_{q(i)}^{3}+J_{q(o)}^{3}+J_{g(i)}^{3}+J_{g(o)}^{3}\right]\right| P S\right\rangle= \pm \frac{1}{2},
$$

where $\mathcal{N}=2(2 \pi)^{3} P^{+} \delta^{3}(0)$. Thus we arrive at the sum rule obeyed by the helicity distribution functions

$$
\int_{0}^{1} d x\left[\Delta q\left(x, Q^{2}\right)+\Delta q_{L}\left(x, Q^{2}\right)+\Delta g\left(x, Q^{2}\right)+\Delta g_{L}\left(x, Q^{2}\right)\right]= \pm \frac{1}{2}
$$

as a result of light-front helicity conservation.

\section{E. Sum rule for $g_{T}$ and the Burkhardt-Cottingham sum rule}

From Eq.(4.15) it follows that

$$
\int_{0}^{1} d x g_{1}\left(x, Q^{2}\right)=\frac{1}{16 \pi} \int_{-\infty}^{+\infty} d x \int d \eta e^{-\eta x} \sum_{\alpha} e_{\alpha}^{2}\left(\bar{A}_{1 \alpha}+\frac{1}{2} P^{+} \xi^{-} \bar{A}_{2 \alpha}\right) .
$$

Provided the bilocal form factor $\bar{A}_{2 \alpha}$ does not have pathological behavior as $\xi^{-} \rightarrow 0$, we have,

$$
\int_{0}^{1} d x g_{1}\left(x, Q^{2}\right)=\frac{1}{8} \bar{A}_{1 \alpha}(0) .
$$

Also we have, from Eq.(4.17),

$$
\int_{0}^{1} d x g_{T}\left(x, Q^{2}\right)=\frac{1}{8} \bar{A}_{1 \alpha}(0) .
$$

Since, $g_{T}=g_{1}+g_{2}$, it follows from Eqs. 6.42) and (6.43) that

$$
\int_{0}^{1} d x g_{2}\left(x, Q^{2}\right)=0
$$

which is the Burkhardt-Cottingham sum rule.

Recently, in the literature, there have been discussions about the validity of BC sum rule

in perturbative QCD [24. Here we have shown the validity of the sum rule exactly up to the leading contribution in the $1 / q^{-}$expansion without recourse to perturbation theory. 
Obviously, the BC sum rule does not provide us any intuition about the physical picture of $g_{2}$. Indeed, as we have pointed out the physical picture for $g_{2}$ is not clear, since experimentally one directly measures $g_{1}$ and $g_{T}$ when the target is polarized in the longitudinal and transverse directions, respectively. The transverse polarized structure function is $g_{T}$ rather than $g_{2}$. Eqs. (6.42) and (6.43) indicates that by averaging over $x$, the longitudinal and the transverse structure functions give the same result. This can be again regarded as a consequence of rotational symmetry. However, this does not implies that $g_{1}(x)$ and $g_{T}(x)$ are the same.

To see clearly the intrinsic physical picture of $g_{T}\left(x, Q^{2}\right)$, let us consider the target state being transversely polarized in the $x$-direction without loss of generality. Then we can simply express $|P S\rangle$ as a combination of the helicity up and down states: $\left|P S_{\perp}^{x}\right\rangle=\frac{1}{\sqrt{2}}(|P \uparrow\rangle \pm|P \downarrow\rangle)$ for $S^{x}= \pm M$. It is easy to show that $g_{T}$ measures the helicity flip processes on the light-front [20],

$$
\begin{gathered}
g_{T}\left(x, Q^{2}\right)=\frac{1}{8 \pi M} \int_{-\infty}^{\infty} d \eta e^{-i \eta x} \frac{1}{2} \sum_{\lambda}\langle P \lambda| \bar{\psi}_{\alpha}\left(\xi^{-}\right)\left(\gamma^{i}-\frac{P^{i}}{P^{+}} \gamma^{+}\right) \gamma_{5} \psi_{\alpha}(0) \\
+h . c|P-\lambda\rangle
\end{gathered}
$$

Not that the quantity $g_{2}$ is purely introduced in the Lorentz decomposition of the hadronic tensor $W^{\mu \nu}$ for historical reasons and has no clear physical interpretation. Only $g_{1}$ and $g_{T}$ have the clear physical picture: $g_{1}$ measures the parton helicity distribution and $g_{T}$ measures the parton helicity flip effect, which is equivalent to the measurement of the effects of chiral symmetry breaking and therefore it involves more complicated intrinsic dynamics of quarks and gluons. A possible relation between $g_{T}$ and dynamical chiral symmetry breaking is explored in [20], and more detailed theoretical and experimental investigations remain to be further carried out.

\section{F. Sum rule for twist four part of $F_{L}$}

¿From Eqs. (4.12) and (4.14) it follows that $F_{2}(-x)=F_{2}(x)$ and $F_{L}^{\tau=4}(-x)=-F_{L}^{\tau=4}(x)$. Consider the integral

$$
\begin{aligned}
\int_{-\infty}^{+\infty} d x \frac{F_{L}^{\tau=4}(x)}{x} & =2 \int_{0}^{\infty} d x \frac{F_{L}^{\tau=4}(x)}{x} \\
= & \frac{P^{+}}{\pi Q^{2}} \int_{-\infty}^{+\infty} x d x \int d \eta e^{-i \eta x} \sum_{\alpha} e_{\alpha}^{2}\langle P| \bar{\psi}_{\alpha}\left(\xi^{-}\right)\left(\gamma^{-}\right. \\
& \left.\left.-\frac{\left(P_{\perp}\right)^{2}}{\left(P^{+}\right)^{2}}\right) \psi_{\alpha}(0)-\text { h.c. }|P\rangle\right\}
\end{aligned}
$$

where in the first equality, the symmetry property of $F_{L}^{\tau=4}$ has been used. Interchanging the orders of $x$ and $y^{-}$integrations and carrying out the integrations explicitly, we arrive at [25]

$$
\int_{0}^{1} d x \frac{F_{L}^{\tau=4}\left(x, Q^{2}\right)}{x}=\frac{2}{Q^{2}} \sum_{\alpha} e_{\alpha}^{2}\left[\left\langle P\left|\bar{\psi}_{\alpha}(0) i\left(\gamma^{-} \partial^{+}-\frac{\left(P_{\perp}\right)^{2}}{\left(P^{+}\right)^{2}} \gamma^{+} \partial^{+}\right) \psi_{\alpha}(0)\right| P\right\rangle\right] .
$$


Identifying $i \bar{\psi} \gamma^{-} \partial^{+} \psi=\theta_{q}^{+-}$, the fermionic part of the light-front QCD Hamiltonian density and $i \bar{\psi} \gamma^{+} \partial^{+} \psi=\theta_{q}^{++}$, the fermionic part of the light-front QCD longitudinal momentum density, (see Eqs. (6.4) and (6.13) above), we arrive at the interesting relation:

$$
\int_{0}^{1} d x \frac{F_{L}^{\tau=4}\left(x, Q^{2}\right)}{x}=\frac{2}{Q^{2}} \sum_{\alpha} e_{\alpha}^{2}\left[\langle P|\left(\theta_{q \alpha}^{+-}(0)-\frac{\left(P_{\perp}\right)^{2}}{\left(P^{+}\right)^{2}} \theta_{q \alpha}^{++}(0)|P\rangle\right],\right.
$$

We have used the fact that the physical structure function vanishes for $x>1$. We observe that the integral of $\frac{F_{L(q)}^{\tau=4}}{x}$ is related to the hadron matrix element of the fermionic parts of the light-front Hamiltonian density. The above relation makes manifest the non-perturbative nature of the twist-four part of the longitudinal structure function.

The fermionic operator matrix elements appearing in Eq. (6.48) changes with $Q^{2}$ as a result of the mixing of quark and gluon operators in QCD under renormalization. Next we analyze this problem of operator mixing and derive a new sum rule at the twist four level arising as a result of the conservation of energy-momentum tensor.

We define the twist four longitudinal gluon structure function

$$
\begin{aligned}
F_{L(g)}^{\tau=4}(x)= & \frac{1}{Q^{2}} \frac{x P^{+}}{2 \pi} \int d \xi^{-} e^{-i \eta x} \\
& {\left[\left\langle P\left|(-) F^{+\lambda a}\left(\xi^{-}\right) F_{\lambda a}^{-}(0)+\frac{1}{4} g^{+-} F^{\lambda \sigma a}\left(\xi^{-}\right) F_{\lambda \sigma a}(0)\right| P\right\rangle\right.} \\
& \left.\quad-\frac{\left(P^{\perp}\right)^{2}}{\left(P^{+}\right)^{2}}\left\langle P\left|F^{+\lambda a}\left(\xi^{-}\right) F_{\lambda a}^{+}(0)\right| P\right\rangle\right] .
\end{aligned}
$$

Then if we assume $e_{\alpha}=1$, we have,

$$
\int_{0}^{1} \frac{d x}{x}\left[F_{L}^{\tau=4}+F_{L(g)}^{\tau=4}\right]=\frac{2}{Q^{2}}\left[\left\langle P\left|\theta^{+-}(0)\right| P\right\rangle-\frac{\left(P^{\perp}\right)^{2}}{\left(P^{+}\right)^{2}}\left\langle P\left|\theta^{++}(0)\right| P\right\rangle\right] .
$$

But,

$$
\left\langle P\left|\theta^{+-}(0)\right| P\right\rangle=2 P^{+} P^{-}=2\left(M^{2}+\left(P^{\perp}\right)^{2}\right) \text { and }\left\langle P\left|\theta^{++}(0)\right| P\right\rangle=2\left(P^{+}\right)^{2},
$$

where $M$ is the invariant mass of the hadron. Thus we arrive at a new sum rule for the twist four part of the longitudinal structure function [25]

$$
\int_{0}^{1} \frac{d x}{x}\left(F_{L}^{\tau=4}+F_{L(g)}^{\tau=4}\right)=4 \frac{M^{2}}{Q^{2}}
$$

\section{CONCLUSION}

In this paper, beginning with an inverse power expansion of the light-front energy of the probe in the framework of light-front $\mathrm{QCD}$, we have arrived at the most general expression for the leading contributions to deep inelastic structure functions as the Fourier transform of the matrix element of different components of bilocal vector and axial vector currents. Although some of the expressions are already known, others are either completely new, such as the 
expression for $F_{2}$ in terms of the transverse component of bilocal current matrix element and the expression for $F_{L}$, or generalizations of earlier results in some specific Lorentz frame to an arbitrary Lorentz frame, e.g. the expressions for $g_{T}$ (or $\left.g_{2}\right)$.

We have also provided a consistent and Lorentz invariant definition of twist based on light-front power counting. Using the light-front power counting, we found that quark-gluon coupling operators contributing to $g_{2}$ are indeed twist-two when we look at their Lorentz invariant matrix elements. Only $F_{L}$ has the leading twist-four contribution. No twist-three Lorentz invariant matrix element emerges in the leading contributions of the inclusive deep inelastic lepton-nucleon scatterings. The light-front power counting [5] naturally separates the space-time dimensions into two different scales measured in deep inelastic processes, the transverse scale and the longitudinal scale. Only the transverse scale has the mass dimension which determines the $1 / Q^{2}$ suppressions. Therefore the light-front mass dimension seems to be more meaningful than the concept of twist in the analysis of the $1 / Q^{2}$ suppressions. Lightfront power counting analysis may eliminate some confusions in the discussion of higher-twist contributions to structure functions, especially, the transverse polarized structure function.

We have also derived new sum rules for $g_{T}$ and $F_{L}$, which provide the physical picture of these structure functions. An important feature of the present formulation of deep inelastic processes is the fact that we have unified the treatment of soft and hard contributions to the structure functions in terms of multi-parton wave functions. The hard contributions can be easily calculated from light-front time-ordered perturbative expansion in high energy QCD [1.3. 4], while the soft contributions can be evaluated by the multi-parton wave functions below the factorization scale $(\approx 1 \mathrm{GeV})$, by solving the light-front bound state equation based on the recently developed nonperturbative renormalization group approach [5 77 or other approaches on light-front QCD [26]. Further investigations along this direction are in progress and will be published in forthcoming papers.

\section{ACKNOWLEDGMENTS}

We acknowledge enlightening discussions with Stan Brodsky and James Vary. WMZ also thanks H. Y. Cheng, C. Y. Cheung and H. L. Yu for fruitful discussions. This work is partially supported by NSC86-2816-M001-009R-L and NSC86-2112-M001-020 (WMZ). 


\section{REFERENCES}

[1] A. Harindranath, R. Kundu and W. M. Zhang, Deep Inelastic Structure Functions in Light-Front QCD: Radiative Corrections, preprint May, 1998.

[2] G. P. Lepage and S. J. Brodsky, Phys. Rev. D22, 2157 (1980).

[3] W. M. Zhang and A. Harindranath, Phys. Rev. D48, 4868; 4881; A. Harindranath and W. M. Zhang, ibid, 4903 (1993).

[4] A. Harindranath and R. Kundu, preprint (1996) hep-ph/9606433.

[5] K. G. Wilson, T. Walhout, A. Harindranath, W. M. Zhang, R. J. Perry, and St. D. Glazek, Phys. Rev. D49, 6720 (1994).

[6] W. M. Zhang, Phys. Rev. D56, 1528 (1997).

[7] M. M. Brisudova, R. J. Perry and K. G. Wilson, Phys. Rev. Lett. 78, 1227 (1997).

[8] R. P. Feynman, Photon Hadron Interactions (W. A. Benjamin, New York, 1972).

[9] See for example, T. Muta, Foundations of Quantum Chromodynamics (World Scientific, Singapore, 1987).

[10] J. Collins, D. Soper and G. Sterman, in Perturbative Quantum Chromo Dynamics, ed. by A. Mueller (World Scientific, Singapore 1989) p.1.

[11] R. Jackiw, Diverse Topics in Theoretical and Mathematical Physics, (World Scientific, Singapore, 1995), p.309.

[12] A. Harindranath and W. M. Zhang, Phys. Lett. B 390, 359 (1997).

[13] R.L. Jaffe and X. Ji, Nucl. Phys. B 375, 527 (1992); R. L. Jaffe, "Spin, Twist, and Hadron Structure in Deep Inelastic Scattering", MIT preprint MIT-CTP-2506, January 1996.

[14] R.K. Ellis, W. Furmanski, and R. Petronzio, Nucl. Phys. B212, 29 (1983); J. Qiu, Phys. Rev. D 42, 30 (1990).

[15] S. J. Brodsky and P. Lepage, in "Quantum Chromodynamics" (A.Mosher, Ed), Proceedings of Summer Institute on Particle Physics, July 9-20, 1979, SLAC Report No. 224, (1980).

[16] S. J. Brodsky et al, Nucl. Phys. B369, 519, (1992).

[17] S. Wandzura and F. Wilczek, Phys. Lett. B72, 195 (1977).

[18] E. V. Shuryak and A. I. Vainshtein, Nucl. Phys. B201, 141 (1982).

[19] A. Harindranath and W. M. Zhang, Phys. Lett. B 408, 347 (1997).

[20] W. M. Zhang and A. Harindranath, preprint (1996) hep-ph/9606347.

[21] H. Y. Cheng, Int. J. Mod. Phys. A11, 5109 (1996).

[22] H. J. Melosh, Phys. Rev. D9, 1095 (1975).

[23] R. L. Jaffe, Phys. Lett. B365, 359 (1996).

[24] R. Mertig and W. L. van Neeven, Z. Phys. C60, 489 (1993); erratum, ibid. C65 (1995) 360; G. Altarelli, B. Lampe, P. Nason and G. Ridolfi, Phys. Lett. B334, 187 (1994).

[25] A. Harindranath, R. Kundu, A. Mukherjee and J. P. Vary, Phys. Lett. B417, 361 (1998), hep-ph/9711298.

[26] S. J. Brodsky, H. C. Pauli and S. S. Pinsky, "Quantum Chromodynamics and Other Field Theories on Light Cone", SLAC-PUB-7484, hep-ph/9705477 and references therein. 